\title{
Finite-temperature exact diagonalization cluster dynamical mean-field study of the two-dimensional Hubbard model: Pseudogap, non-Fermi-liquid behavior, and particle-hole asymmetry
}

\author{
Ansgar Liebsch ${ }^{1}$ and Ning-Hua Tong ${ }^{2}$ \\ ${ }^{1}$ Institut für Festkörperforschung, Forschungszentrum Jülich, 52425 Jülich, Germany \\ ${ }^{2}$ Department of Physics, Renmin University of China, 100872 Beijing, China \\ (Received 16 July 2009; revised manuscript received 10 August 2009; published 27 October 2009)
}

\begin{abstract}
The effect of doping in the two-dimensional Hubbard model is studied within finite-temperature exact diagonalization combined with cluster dynamical mean-field theory. By employing a mixed basis involving cluster sites and bath molecular orbitals for the projection of the lattice Green's function onto $2 \times 2$ clusters, a considerably more accurate description of the low-frequency properties of the self-energy is achieved than in a pure site picture. To evaluate the phase diagram, the transition from Fermi-liquid to non-Fermi-liquid behavior for decreasing hole doping is studied as a function of Coulomb energy, next-nearest-neighbor hopping, and temperature. The self-energy component $\Sigma_{X}$ associated with $X=(\pi, 0)$ is shown to develop a collective mode above $E_{F}$, whose energy and strength exhibits a distinct dispersion with doping. This low-energy excitation gives rise to non-Fermi-liquid behavior as the hole doping decreases below a critical value $\delta_{c}$, and to an increasing particle-hole asymmetry, in agreement with recent photoemission data. This behavior is consistent with the removal of spectral weight from electron states above $E_{F}$ and the opening of a pseudogap, which increases with decreasing doping. The phase diagram reveals that $\delta_{c} \approx 0.15 \ldots 0.20$ for various system parameters. For electron doping, the collective mode of $\Sigma_{X}(\omega)$ and the concomitant pseudogap are located below the Fermi energy, which is consistent with the removal of spectral weight from the hole states just below $E_{F}$. The critical doping, which marks the onset of non-Fermi-liquid behavior, is systematically smaller than for hole doping.
\end{abstract}

DOI: 10.1103/PhysRevB.80.165126

PACS number(s): 71.20.Be

\section{INTRODUCTION}

The nature of the metal-insulator transition as a function of doping is one of the key issues in strongly correlated materials. ${ }^{1}$ Experimental studies of many high- $T_{c}$ cuprates reveal a rich phase diagram, with conventional Fermi-liquid behavior in overdoped metals and an anomalous pseudogap phase in underdoped systems close to the Mott insulator. One of the most intriguing and challenging aspects of the nonFermi-liquid phase is the observation of highly nonisotropic behavior in momentum space. ${ }^{2}$ Whereas along the nodal direction $\Gamma M$ well-defined quasiparticles exist, in the vicinity of $X=(\pi, 0)$ strong deviations from Fermi-liquid behavior occur. In particular, below a critical doping a pseudogap appears, which becomes more prominent close to the Mott insulator. This transition from Fermi-liquid to non-Fermiliquid properties has been widely investigated in recent years, and several theoretical models have been proposed. ${ }^{3-16}$

Dynamical mean-field theory ${ }^{17-22}$ (DMFT) provides an elegant and successful framework for the description of the correlation-induced transition from metallic to Mott insulating behavior. ${ }^{23}$ The local or single-site version of DMFT, however, focuses exclusively on dynamical correlations, which can give rise to spectral weight transfer between low and high frequencies. To address the momentum dependence of the self-energy, it is important to allow for spatial fluctuations, at least on a short-range atomic scale. For this purpose, several approaches based on cluster extensions of DMFT (Refs. 24-27) as well as cluster perturbation theory ${ }^{28}$ have been proposed. The general consensus that has emerged from many studies in this field ${ }^{29-66}$ is that scattering processes are indeed much stronger close to $(\pi, 0)$ and $(0, \pi)$ than in other regions of the Brillouin zone (BZ). Thus, Fermi-liquid behavior first breaks down in the antinodal direction and a pseudogap in the density of states (DOS) opens up. In the nodal direction between $(0,0)$ and $(\pi, \pi)$ Fermi-liquid behavior persists and well-defined quasiparticles can be identified.

In the present work we use exact diagonalization ${ }^{67}$ (ED) in combination with cellular DMFT (Ref. 26) (CDMFT) to investigate the two-dimensional (2D) Hubbard model on a square lattice for $2 \times 2$ clusters. For computational reasons, ED has previously been applied to study this model at $T=0 .{ }^{41,45,46,50,63}$ Here, we employ an extension to finite temperatures by making use of the Arnoldi algorithm, ${ }^{68}$ which provides a highly efficient evaluation of excited states. In view of the approximate nature of quantum impurity solvers, a scheme that complements existing alternative approaches at finite $T$ is clearly desirable. An important advantage of ED is the accessibility of large Coulomb energies and low temperatures, and the absence of sign problems. Moreover, the cluster ED/DMFT is formulated here in terms of a mixed basis involving cluster sites and bath molecular orbitals, thereby allowing a very accurate projection of the lattice Green's function onto the $2 \times 2$ cluster. Thus, despite the use of only two bath levels per cluster orbital (12 levels in total), due to the indirect Coulomb coupling between baths, the spacing between excitation energies is very small so that finite-size errors are greatly reduced even at low temperatures. As a result of these refinements, extrapolation from the Matsubara axis yields highly reliable self-energies and Green's functions at low real frequencies. Preliminary calculations based on this approach were published in Ref. 69. 
Also, this scheme has recently been used to evaluate the phase diagram of the partially frustrated Hubbard model for triangular lattices. ${ }^{70}$

The purpose of this paper is twofold: first, to demonstrate the accuracy of our approach and to illustrate the consistency with previous work, a variety of results are given in detail, in particular, for the cluster self-energy. The excellent overall agreement with results obtained via alternative cluster methods serves to establish finite-temperature cluster ED/DMFT as a versatile and efficient complementary scheme. Second, several aspects of the 2D Hubbard model are studied systematically in order to evaluate the phase diagram for hole and electron doping. The focus here is on the transition from Fermi-liquid to non-Fermi-liquid behavior. In particular, we investigate how the nature of this transition varies as a function of Coulomb energy, next-nearest-neighbor (NNN) hopping, and temperature. A systematic study of this dependence has, to our knowledge, not been carried out before. In addition, we discuss a variety of other aspects such as the comparison between CDMFT and the dynamical cluster approximation $^{27}$ (DCA), the difference between electron and hole doping, the doping variation of the pseudogap and of the weight of induced in-gap states, the approximate momentum variation of the pseudogap, and the particle-hole asymmetry of the spectral function, which has recently been observed in angle-resolved photoemission (ARPES) data for $\mathrm{Bi}_{2} \mathrm{Sr}_{2} \mathrm{CaCu}_{2} \mathrm{O}_{8+\delta}$ by Yang et al. ${ }^{71}$

The key quantity, which exhibits the change from Fermiliquid to non-Fermi-liquid behavior most clearly, is the selfenergy component $\Sigma_{X}$ associated with $X=(\pi, 0)$. For hole doping $\delta \leq 15 \ldots 20 \%$, we show that spatial fluctuations within the cluster give rise to a low-energy collective mode in $\operatorname{Im} \Sigma_{X}(\omega)$, whose energy and strength exhibit a characteristic variation with doping. The real part of $\Sigma_{X}(\omega)$ then exhibits a positive slope above $E_{F}$, implying the removal of spectral weight from electron states and the opening of a pseudogap in the density of states. A resonance in $\operatorname{Im} \Sigma_{X}(\omega)$ at $\delta=0.05$ had also been found by Jarrell et al. ${ }^{30}$ in DCA quantum Monte Carlo (QMC) calculations, and by Kyung et $a l^{46}$ in ED/CDMFT calculations at $T=0$. Here we show that the evolution of this correlation-induced collective mode with decreasing doping leads to the widening of the pseudogap until it merges with the Mott gap at half-filling. As a result, the density of states in this region acquires a very asymmetric shape. At large doping the Fermi level is located at a peak in the density of states, and quasiparticle broadening varies quadratically near $E_{F}$. For decreasing doping the pseudogap shifts downward, giving rise to a marked particlehole asymmetry in the spectral distributions $A(\mathbf{k}, \omega)$ due to enhanced quasiparticle damping predominantly above $E_{F}$. Moreover, with decreasing doping the pseudogap appears first along the antinodal direction before it opens across the entire Fermi surface. The phase diagram shows that the change from Fermi-liquid to non-Fermi-liquid behavior is remarkably stable, $\delta_{c} \approx 0.15 \ldots 0.20$, when system parameters such as Coulomb energy, temperature, or second-neighbor hopping are varied.

For electron doping, the low-energy collective mode is located below the Fermi energy, as expected for the removal of hole states just below $E_{F}$. The doping, which defines the onset of non-Fermi-liquid behavior, is systematically smaller than for hole doping. Finally, the Mott transition induced by electron doping exhibits hysteresis behavior consistent with a first-order transition. In the case of hole doping, hysteresis behavior could not be identified at the temperatures considered in this paper. Thus, within the accuracy of our ED/ CDMFT approach, this transition is either weakly first order at very low temperatures or continuous.

The outline of this paper is as follows. Section II presents the main theoretical aspects of our finite- $T$ cluster ED/DMFT approach. Section III provides the results for $2 \times 2$ clusters. In particular, we discuss the Mott transition, the non-Fermiliquid properties, the comparison between CDMFT and DCA, the pseudogap, the doping variation of the low-energy collective mode, electron doping, the phase diagram, the momentum dependence, the comparison with the dual Fermion method, and the interpretation of recent ARPES measurements. A summary is presented in Sec. IV.

\section{CLUSTER ED/DMFT IN MIXED SITE/ORBITAL BASIS}

In this section we outline the finite-temperature ED method in the mixed site/molecular-orbital basis, which is employed as highly efficient and accurate impurity solver in the cluster DMFT. Let us consider the single-band Hubbard model for a two-dimensional square lattice

$$
H=-\sum_{\langle i j\rangle \sigma} t_{i j}\left(c_{i \sigma}^{+} c_{j \sigma}+\text { H.c. }\right)+U \sum_{i} n_{i \uparrow} n_{i \downarrow},
$$

where the sum in the first term extends up to second neighbors. The band dispersion is given by $\boldsymbol{\epsilon}(\mathbf{k})=-2 t\left[\cos \left(k_{x}\right)\right.$ $\left.+\cos \left(k_{y}\right)\right]-4 t^{\prime} \cos \left(k_{x}\right) \cos \left(k_{y}\right)$. In order to approximately represent hole-doped cuprate systems, the nearest-neighbor hopping integral is defined as $t=0.25$ (bandwidth $W=2$ ). The next-nearest-neighbor integral is mainly defined as $t^{\prime}=$ $-0.3 t=-0.075$, but $t^{\prime}=0$ will also be considered. The local Coulomb interaction is taken to be $U=10 t=2.5$ and $U=6 t$ $=1.5$. Thus, at half-filling, the system is a Mott insulator. (For $t^{\prime}=0$, QMC/DMFT calculations for four-site clusters ${ }^{55}$ yield $U_{c} \approx 1.4 \ldots 1.5$, in agreement with ED/DMFT results for two-site and four-site clusters. ${ }^{69}$ These values are consistent with recent $\mathrm{QMC} / \mathrm{DCA}$ calculations for eight-site clusters $^{66}$ which give $U_{c} \approx 1.4 \ldots 1.6$.)

Within CDMFT (Ref. 26) the interacting lattice Green's function in the cluster site basis is given by

$$
G_{i j}\left(i \omega_{n}\right)=\sum_{\mathbf{k}}\left[i \omega_{n}+\mu-t(\mathbf{k})-\Sigma\left(i \omega_{n}\right)\right]_{i j}^{-1},
$$

where the $\mathbf{k}$ sum extends over the reduced Brillouin Zone, $\omega_{n}=(2 n+1) \pi T$ are Matsubara frequencies, and $\mu$ is the chemical potential. $t(\mathbf{k})$ denotes the hopping matrix for the superlattice and $\Sigma_{i j}\left(i \omega_{n}\right)$ represents the cluster self-energy matrix. The lattice constant is taken to be $a=1$ and site labels refer to $1 \equiv(0,0), 2 \equiv(1,0), 3 \equiv(0,1)$, and $4 \equiv(1,1)$. In this geometry, all diagonal elements of the symmetric matrix $G_{i j}$ are identical and there are only two independent off-diagonal elements: $G_{12}=G_{13}=G_{24}=G_{34}$ and $G_{14}=G_{23}$. By definition, both the lattice Green's function $G_{i j}$ and self-energy $\Sigma_{i j}$ have 


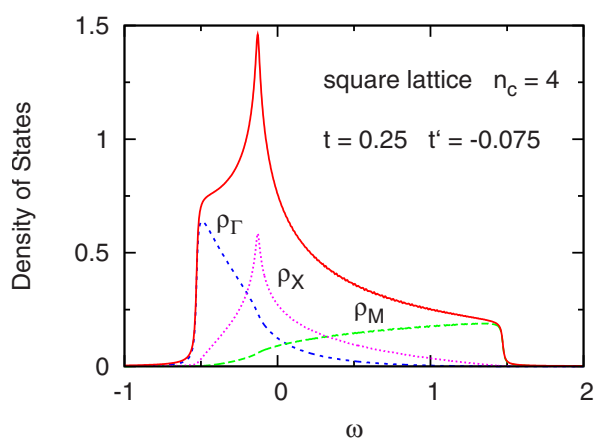

FIG. 1. (Color online) Total DOS $\rho(\omega)$ and molecular-orbital components $\rho_{m}(\omega)$ for four-site clusters of square lattice. For clarity, the molecular-orbital components are divided by $n_{c}=4$. $\omega=0$ defines the Fermi energy for half-filling. At $14 \%$ hole doping the van Hove singularity is shifted from $\omega=-0.13$ to -0.09 .

continuous spectral distributions at real $\omega$. Only the paramagnetic phase will be considered here.

It is useful to transform the site basis into a molecularorbital basis in which the Green's function and self-energy become diagonal. The orbitals are defined as: $\phi_{1}=(|1\rangle+|2\rangle$ $+|3\rangle+|4\rangle) / 2, \quad \phi_{2}=(|1\rangle-|2\rangle-|3\rangle+|4\rangle) / 2, \quad \phi_{3}=(|1\rangle+|2\rangle-|3\rangle$ $-|4\rangle) / 2$, and $\phi_{4}=(|1\rangle-|2\rangle+|3\rangle-|4\rangle) / 2$. We refer to these orbitals as $\Gamma, M$, and $X$, respectively, where $X$ is doubly degenerate. The Green's function elements in this basis will be denoted as $G_{m}\left(i \omega_{n}\right)$, where

$$
\begin{gathered}
G_{\Gamma} \equiv G_{1}=G_{11}+2 G_{12}+G_{14}, \\
G_{M} \equiv G_{2}=G_{11}-2 G_{12}+G_{14}, \\
G_{X} \equiv G_{3}=G_{4}=G_{11}-G_{14} .
\end{gathered}
$$

An analogous notation is used for the self-energy. Similar diagonal representations of $G$ and $\Sigma$ have been used in several previous works. $30,41,54,55,59,60,69,72$

Figure 1 illustrates the uncorrelated density of states components in the molecular-orbital basis, where $\rho_{m}(\omega)=$ $-\frac{1}{\pi} \operatorname{Im} G_{m}(\omega)$ for $\Sigma=0$, and we denote $\rho_{\Gamma}=\rho_{1}, \rho_{M}=\rho_{2}$, and $\rho_{X}=\rho_{3,4}$. The average or local density is $\rho_{a v}=\left(\rho_{\Gamma}+\rho_{M}\right.$ $\left.+2 \rho_{X}\right) / 4$. Note that all molecular-orbital densities extend across the entire bandwidth. Nevertheless, only $\rho_{X}$ contains the van Hove singularity, while $\rho_{\Gamma}$ and $\rho_{M}$ are roughly representative of the spectral weight near $\mathbf{k}=(0,0)$ and $\mathbf{k}$ $=(\pi, \pi)$, respectively. Hole doping shifts the van Hove singularity toward $E_{F}$, whereas electron doping moves this singularity away from $E_{F}$.

A central feature of DMFT is that, to avoid double counting of Coulomb interactions in the quantum impurity calculation, the self-energy must be removed from the small cluster in which correlations are treated explicitly. This removal yields the Green's function

$$
G_{0}\left(i \omega_{n}\right)=\left[G\left(i \omega_{n}\right)^{-1}+\Sigma\left(i \omega_{n}\right)\right]^{-1},
$$

which is also diagonal in the molecular-orbital basis.

For the purpose of performing the ED calculation we now project the diagonal components of $G_{0}\left(i \omega_{n}\right)$ onto those of a larger cluster consisting of $n_{c}=4$ impurity levels and $n_{b}=8$ bath levels. The total number of levels is $n_{s}=n_{c}+n_{b}=12$. Thus,

$$
G_{0, m}\left(i \omega_{n}\right) \approx G_{0, m}^{c l}\left(i \omega_{n}\right)=\left(i \omega_{n}+\mu-\epsilon_{m}-\sum_{k=5}^{12} \frac{\left|V_{m k}\right|^{2}}{i \omega_{n}-\epsilon_{k}}\right)^{-1},
$$

where $\epsilon_{m}$ denote the molecular-orbital levels, $\epsilon_{k}$ denote the bath levels, and $V_{m k}$ denote the hybridization matrix elements. The incorporation of the impurity level $\epsilon_{m}$ ensures a much better fit of $G_{0, m}\left(i \omega_{n}\right)$ than by projecting only onto bath orbitals.

Assuming independent baths for the cluster orbitals, each component $G_{0, m}\left(i \omega_{n}\right)$ is fitted using five parameters: one impurity level $\epsilon_{m}$, two bath levels $\epsilon_{k}$, and two hopping integrals $V_{m k}$. For instance, orbital 1 couples to bath levels 5 and 9 , orbital 2 couples to bath levels 6 and 10, etc. For the three independent cluster Green's functions, we, therefore, use a total of 15 fit parameters to represent $G_{0}\left(i \omega_{n}\right)$. This procedure provides a considerably more flexible projection than within a pure site basis. Since for symmetry reasons all sites are equivalent, one would have in this case only four parameters (without including a level at the cluster sites). Thus, the molecular-orbital basis allows for 11 additional cross hybridization terms as well as internal cluster couplings (see below). In addition, it is much more reliable to fit the three independent molecular-orbital components $G_{0, m}\left(i \omega_{n}\right)$ than a nondiagonal site matrix $G_{0, i j}\left(i \omega_{n}\right)$ with only four parameters.

Figure 2 illustrates the projection of the lattice Green's function $G_{0}\left(i \omega_{n}\right)$ onto the cluster for $U=2.5$ and $\mu=0.5$, which corresponds to about $\delta=0.08$ hole doping. Projections of similar quality are achieved at other Coulomb energies and chemical potentials.

The evaluation of the finite-temperature interacting cluster Green's function could in principle also be carried out in the molecular-orbital basis. The Coulomb interaction then becomes a matrix containing many interorbital components. This step can be circumvented by using a mixed basis consisting of cluster sites $i$ and bath molecular orbitals $k$. Thus, the diagonal $8 \times 8$ subblock $h_{b}=\left(\epsilon_{k} \delta_{k k^{\prime}}\right)$ representing the bath levels remains unchanged, but the diagonal $4 \times 4$ cluster molecular-orbital submatrix now becomes nondiagonal in the cluster site basis. The transformation between sites $i$ and orbitals $m$ is given by

$$
T_{i m}=0.5\left(\begin{array}{cccc}
1 & 1 & 1 & 1 \\
1 & -1 & -1 & 1 \\
1 & -1 & 1 & -1 \\
1 & 1 & -1 & -1
\end{array}\right) \text {. }
$$

In this mixed basis, the site subblock of the cluster Hamiltonian becomes

$$
h_{c}=\left(\begin{array}{cccc}
\epsilon & \tau & \tau & \tau^{\prime} \\
\tau & \epsilon & \tau^{\prime} & \tau \\
\tau & \tau^{\prime} & \epsilon & \tau \\
\tau^{\prime} & \tau & \tau & \epsilon
\end{array}\right)
$$

with $\epsilon=\left(\epsilon_{1}+\epsilon_{2}+2 \epsilon_{3}\right) / 4, \quad \tau=\left(\epsilon_{1}+\epsilon_{2}-2 \epsilon_{3}\right) / 4$, and $\tau^{\prime}=\left(\epsilon_{1}\right.$ $\left.-\epsilon_{2}\right) / 2$. Note that the hopping elements $t$ and $t^{\prime}$ of the origi- 

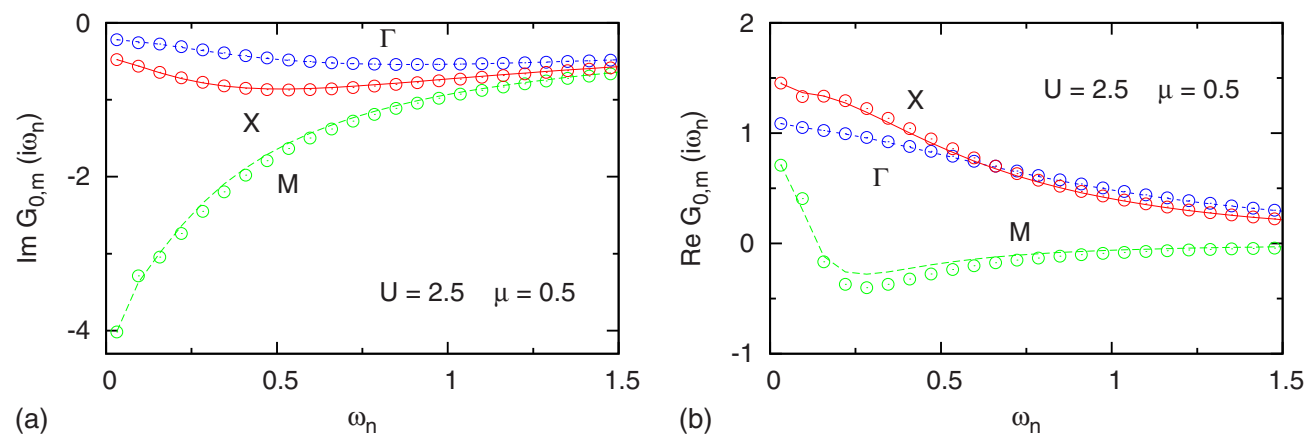

FIG. 2. (Color online) Projection of lattice Green's function components $G_{0, m}\left(i \omega_{n}\right)$ onto cluster consisting of four impurity levels and eight bath levels, for $U=2.5, \mu=0.5$, and $T=0.01$. (a) $\operatorname{Im} G_{0, m}$, (b) $\operatorname{Re} G_{0, m}$. Continuous curves: diagonal elements of lattice Green's function, Eq. (4); circles: approximate expression, right-hand side of Eq. (5).

nal lattice Hamiltonian do not appear since they are effectively absorbed into $\tau$ and $\tau^{\prime}$ via the molecular-orbital cluster levels $\epsilon_{m}$, which are adjusted to fit $G_{0, m}\left(i \omega_{n}\right)$. Evidently, the procedure outlined above not only includes hopping between cluster and bath but also introduces three new parameters within the $2 \times 2$ cluster: $\epsilon, \tau$, and $\tau^{\prime}$. In the mixed basis, the hybridization matrix elements $V_{m k}$ between cluster and bath molecular orbitals introduced in Eq. (5) are transformed to new hybridization matrix elements between cluster sites $i$ and bath orbitals $k$. They are given by

$$
V_{i k}^{\prime}=(T V)_{i k}=\sum_{m} T_{i m} V_{m k}
$$

Thus, the upper right $4 \times 8$ submatrix containing the cluster/ bath hybridization matrix elements is transformed from

$$
\left(\begin{array}{cccccccc}
V_{5} & 0 & 0 & 0 & V_{9} & 0 & 0 & 0 \\
0 & V_{6} & 0 & 0 & 0 & V_{10} & 0 & 0 \\
0 & 0 & V_{7} & 0 & 0 & 0 & V_{11} & 0 \\
0 & 0 & 0 & V_{8} & 0 & 0 & 0 & V_{12}
\end{array}\right)
$$

to

$$
\left(\begin{array}{cccccccc}
V_{5} & V_{6} & V_{7} & V_{8} & V_{9} & V_{10} & V_{11} & V_{12} \\
V_{5} & -V_{6} & -V_{7} & V_{8} & V_{9} & -V_{10} & -V_{11} & V_{12} \\
V_{5} & -V_{6} & V_{7} & -V_{8} & V_{9} & -V_{10} & V_{11} & -V_{12} \\
V_{5} & V_{6} & -V_{7} & -V_{8} & V_{9} & V_{10} & -V_{11} & -V_{12}
\end{array}\right) .
$$

The single-particle part of the cluster Hamiltonian then reads

$$
h_{0}=\left(\begin{array}{cc}
h_{c} & V^{\prime} \\
V^{\prime t} & h_{b}
\end{array}\right) \text {. }
$$

Adding the onsite Coulomb interactions to this Hamiltonian, the nondiagonal interacting cluster Green's function at finite $T$ can be derived from the expression ${ }^{73,74}$

$$
\begin{aligned}
G_{i j}^{c l}\left(i \omega_{n}\right)= & \frac{1}{Z} \sum_{\nu \mu} e^{-\beta E_{\nu}}\left(\frac{\left\langle\nu\left|c_{i \sigma}\right| \mu\right\rangle\left\langle\mu\left|c_{j \sigma}^{+}\right| \nu\right\rangle}{E_{\nu}-E_{\mu}+i \omega_{n}}\right. \\
& \left.+\frac{\left\langle\nu\left|c_{i \sigma}^{+}\right| \mu\right\rangle\left\langle\mu\left|c_{j \sigma}\right| \nu\right\rangle}{E_{\mu}-E_{\nu}+i \omega_{n}}\right)
\end{aligned}
$$

where $E_{\nu}$ and $|\nu\rangle$ denote the eigenvalues and eigenvectors of the Hamiltonian, $\beta=1 / T$ and $Z=\Sigma_{\nu} \exp \left(-\beta E_{\nu}\right)$ is the partition function. At low temperatures only a small number of excited states in a few spin sectors contributes to $G_{i j}^{c l}$. They can be efficiently evaluated using the Arnoldi algorithm. ${ }^{68}$ The excited-state Green's functions are computed using the Lanczos procedure. Further details are provided in Ref. 73. The nondiagonal elements of $G_{i j}^{c l}$ are derived by first evaluating the diagonal components $G_{i i}^{c l}$ and then using the relation

$$
G_{(i+j)(i+j)}^{c l}=G_{i i}^{c l}+G_{i j}^{c l}+G_{j i}^{c l}+G_{j j}^{c l} .
$$

Since $G_{i j}^{c l}=G_{j i}^{c l}$, this yields

$$
G_{i j}^{c l}=\frac{1}{2}\left[G_{(i+j)(i+j)}^{c l}-G_{i i}^{c l}-G_{j j}^{c l}\right] .
$$

The interacting cluster Green's function $G_{i j}^{c l}$ satisfies the same symmetry properties as $G_{i j}$ and $G_{0, i j}$. It may, therefore, also be diagonalized, yielding cluster molecular-orbital components $G_{m}^{c l}$. The cluster molecular-orbital self-energies can then be defined by an expression analogous to Eq. (4)

$$
\Sigma_{m}^{c l}\left(i \omega_{n}\right)=1 / G_{0, m}^{c l}\left(i \omega_{n}\right)-1 / G_{m}^{c l}\left(i \omega_{n}\right) .
$$

At real $\omega$, these cluster self-energy components, just like $G_{0, m}^{c l}$ and $G_{m}^{c l}$, have discrete spectral distributions.

The key assumption in DMFT is now that the impurity cluster self-energy is a physically reasonable representation of the lattice self-energy. Thus,

$$
\Sigma_{m}\left(i \omega_{n}\right) \approx \Sigma_{m}^{c l}\left(i \omega_{n}\right)
$$

where, at real frequencies, $\Sigma_{m}$ is continuous.

In the next iteration step, these diagonal self-energy components are used as input in the lattice Green's function [Eq. (2)], which in the molecular-orbital basis is given by 

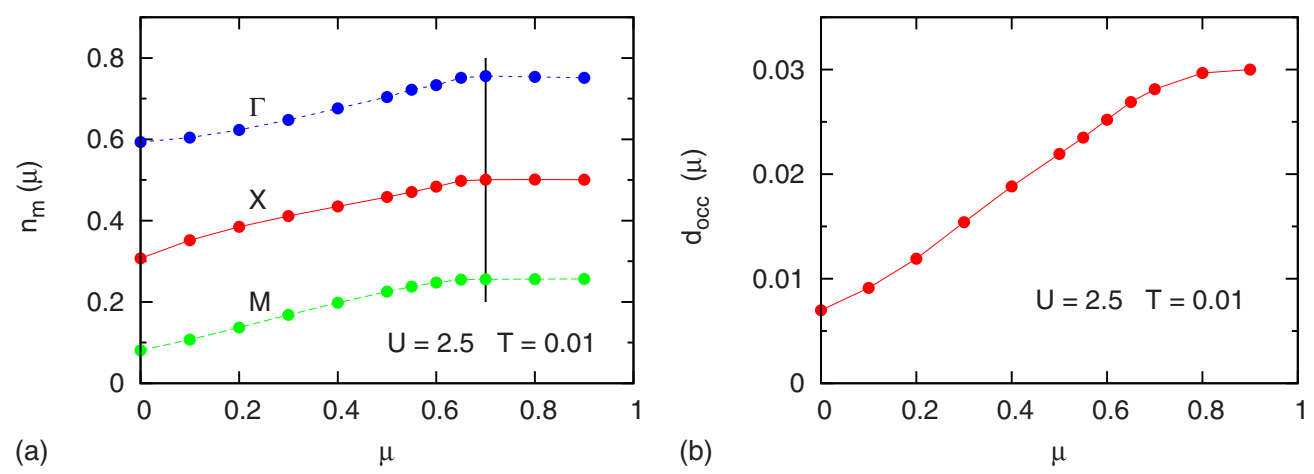

FIG. 3. (Color online) (a) Occupancies of cluster molecular orbitals (per spin) as functions of chemical potential $\mu$, for $U=2.5$, $T=0.01$. The Mott transition occurs at about $\mu \approx 0.7$, indicated by the vertical bar, where $n_{X} \rightarrow 0.5, n_{\Gamma} \rightarrow 0.75$, and $n_{M} \rightarrow 0.25$. (b) Average double occupancy per site as a function of $\mu$.

$$
G_{m}\left(i \omega_{n}\right)=\sum_{\mathbf{k}}\left[i \omega_{n}+\mu-T t(\mathbf{k}) T^{-1}-\Sigma\left(i \omega_{n}\right)\right]_{m m}^{-1},
$$

where $T$ is the transformation defined in Eq. (6). Thus, except for the diagonalization, which is carried out in the mixed site/molecular-orbital basis, all other steps of the calculation procedure are performed in the diagonal orbital basis. Note that $T t(\mathbf{k}) T^{-1}$ is not diagonal at general $\mathbf{k}$ points. As a result, all orbital components of $\Sigma\left(i \omega_{n}\right)$ contribute to each $G_{m}\left(i \omega_{n}\right)$. This feature of CDMFT differs from DCA where one has a one-to-one relation between $\Sigma_{m}\left(i \omega_{n}\right)$ and $G_{m}\left(i \omega_{n}\right)$ (Ref. 27)

$$
G_{m}^{\mathrm{DCA}}\left(i \omega_{n}\right)=\sum_{\mathbf{k}_{m}}\left[i \omega_{n}+\mu-\epsilon(\mathbf{k})-\Sigma_{m}\left(i \omega_{n}\right)\right]^{-1},
$$

where $\mathbf{k}_{m}$ labels the $m$ th patch of the Brillouin zone.

The largest spin sector for $n_{s}=12$ is $n_{\uparrow}=n_{\downarrow}=6$ with dimension $N=853776$. The interacting cluster Hamiltonian matrix $h$ is extremely sparse, so that only about 20 nonzero matrix elements per row need to be stored. Thus, storage requirements are less than for complete diagonalization for $n_{s}=8$. Since the Arnoldi algorithm requires only operations of the type $h u=v$, where $u, v$ are vectors of dimension $N$, the procedure outlined above can easily be parallelized. At temperatures on the order of $T=0.005 \ldots 0.02$, one iteration takes about 15 to $60 \mathrm{~min}$ on eight processors. Except near the Mott transition, five to 10 iterations are usually required to achieve self-consistency. An interesting future development would be the extension to even larger clusters by exploiting massively parallel tools.

We conclude this section by pointing out that, once iteration to self-consistency has been carried out, an approximate periodic lattice Green's function may be constructed from the cluster components in Eq. (2) by using the superposition $^{38}$

$$
\begin{aligned}
G\left(\mathbf{k}, i \omega_{n}\right)= & \frac{1}{4} \sum_{i j=1}^{4} e^{i \mathbf{k} \cdot\left(\mathbf{R}_{i}-\mathbf{R}_{j}\right)} G_{i j}\left(i \omega_{n}\right) \\
= & G_{11}\left(i \omega_{n}\right)+G_{12}\left(i \omega_{n}\right)\left[\cos \left(k_{x}\right)+\cos \left(k_{y}\right)\right] \\
& +G_{14}\left(i \omega_{n}\right) \cos \left(k_{x}\right) \cos \left(k_{y}\right) .
\end{aligned}
$$

At high-symmetry points, this definition coincides with the diagonal elements introduced in Eq. (4). Thus, $G_{\Gamma}\left(i \omega_{n}\right)$ $=G\left[(0,0), i \omega_{n}\right], \quad G_{M}\left(i \omega_{n}\right)=G\left[(\pi, \pi), i \omega_{n}\right], \quad$ and $\quad G_{X}\left(i \omega_{n}\right)$ $=G\left[(\pi, 0), i \omega_{n}\right]=G\left[(0, \pi), i \omega_{n}\right]$. At $\mathbf{k}=(\pi / 2, \pi / 2), G$ coincides with the onsite Green's function $G_{11}=\left(G_{\Gamma}+G_{M}\right.$ $\left.+2 G_{X}\right) / 4$.

\section{RESULTS AND DISCUSSION}

\section{A. Mott transition}

Figure 3 shows the occupancies of the cluster molecular orbitals $\Gamma, M$, and $X$ as functions of chemical potential. The average occupancy per site (both spins) is $n=\left(n_{\Gamma}+n_{M}\right.$ $\left.+2 n_{X}\right) / 2=1-\delta$, where $\delta$ is the hole doping. As revealed by the spectral distributions discussed below, the Mott transition occurs at $\mu \approx 0.7$, where the $X$ orbital becomes half-filled, whereas $n_{\Gamma}$ and $n_{M}$ approach 0.25 and 0.75 , respectively. Thus, all three orbitals take part in the transition. This result is consistent with previous ED/DMFT calculations ${ }^{69}$ for twosite and four-site clusters in the limit $t^{\prime}=0$, and with recent QMC results ${ }^{61}$ for a minimal two-site cluster DCA version, where hole doping takes place at about the same rate for both inner and outer regions of the Brillouin zone. These trends differ, however, from results for an eight-site continuous time QMC/DCA calculation, ${ }^{66}$ which reveals initial doping primarily along the nodal direction, while near $X$ the occupancy for small $\delta$ remains at the same value as in the Mott insulator. Evidently, two-site and four-site cluster DMFT approaches do not provide sufficient momentum resolution to allow for $\mathbf{k}$-dependent doping.

Figure 3(b) shows the average double occupancy per site. We have calculated these occupancies both for increasing and decreasing chemical potential without encountering hysteresis behavior for $T \geq 0.005$. The Mott transition induced by hole doping is, therefore, weakly first order at even lower temperatures, or continuous. This result differs from the case of electron doping discussed farther below, where $n_{m}(\mu)$ as well as $d_{\text {occ }}(\mu)$ readily show hysteresis.

To illustrate the Mott transition in the limit of half-filling, we show in Fig. 4 the spectral distributions obtained from the interacting cluster Green's function: $A_{m}(\omega)=$ $-(1 / \pi) \operatorname{Im} G_{m}^{c l}(\omega+i \gamma)$, where $\gamma=0.02$. These spectra can be evaluated without requiring analytic continuation from Mat- 

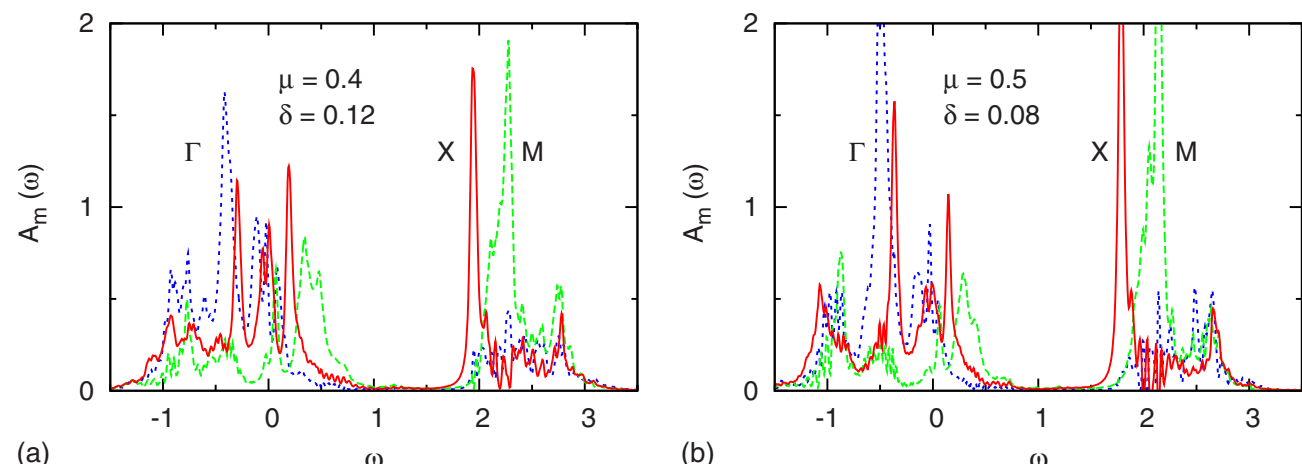

(b)

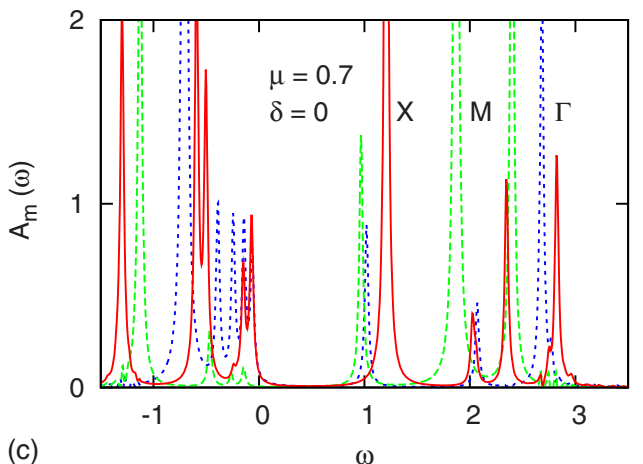

FIG. 4. (Color online) Cluster spectral distributions for various chemical potentials corresponding to hole doping $\delta=0.12, \delta=0.08$, and $\delta=0 ; U=2.5, T=0.01$; broadening $\gamma=0.02$. Solid red curves: $A_{X}(\omega)$; short-dashed blue curves: $A_{\Gamma}(\omega)$, long-dashed green curves: $A_{M}(\omega)$.

subara to real frequencies. The total density of states per spin is given by $A(\omega)=\left[A_{\Gamma}(\omega)+A_{M}(\omega)+2 A_{X}(\omega)\right] / 4$. All cluster molecular orbitals contribute to the spectral weight near the Fermi level in the metallic phase for $\delta>0$, and to the upper and lower Hubbard bands in the Mott phase at $\delta=0$.

The evolution of these spectra as a function of doping supports the picture conjectured long ago by Eskes et al. ${ }^{75}$ Upon hole doping, spectral weight is transferred from the upper and lower Hubbard bands to states just above $E_{F}$, in the lower part of the Mott gap. Since the spectral weight (per spin) of both Hubbard bands initially decreases like (1 $-\delta$ ) $/ 2$, the states induced just above $E_{F}$ have weight $\delta$ (see also Ref. 16). This scenario is a remarkable consequence of strong dynamical correlations and differs fundamentally from the one in ordinary semiconductors, where states induced in the gap have weight $\delta / 2$ per spin for total doping $\delta$.

Our ED/DMFT cluster calculations are in excellent agreement with this picture, as illustrated in Fig. 5, which shows the integrated spectral weight per spin induced just above $E_{F}$. This weight is denoted here as $W_{+}(\delta)$. The initial slope of $W_{+}$ is seen to be well represented by $\delta$, confirming the scenario discussed above. At finite doping $W_{+}(\delta)$ becomes even larger than $\delta+n_{d}$, where $n_{d}$ is the double occupancy shown in Fig. 3. These results differ from those for $t^{\prime}=0$ and $T=0$ obtained by Sakai et al. ${ }^{63}$ who found $W_{+}(\delta) \approx \delta+n_{d}$ up to about $14 \%$ hole doping.

Upon closer inspection, the spectral distributions shown in Fig. 4 at finite doping reveal a pseudogap close to $E_{F}$, which will be discussed in more detail in the following subsections. As shown below, this pseudogap is intimately related to the non-Fermi-liquid properties, which are evident in the $X$ component of the self-energy.

\section{B. Non-Fermi-liquid properties}

We now discuss the low-frequency variation of the cluster self-energy, which is strikingly different for the different cluster molecular orbitals. Figure 6 shows the imaginary parts of $\Sigma_{m}\left(i \omega_{n}\right)$, Eq. (16), for chemical potentials $\mu$ in the range from $1 \%$ to $24 \%$ hole doping. The $\Gamma$ orbital, approximately representative of the center of the Brillouin zone, exhibits the weakest self-energy. It is nearly independent of doping and Fermi-liquid-type, with only a moderate effective-mass enhancement. $\Sigma_{M}$ changes from Fermi-liquid behavior at large doping to insulating behavior $\sim 1 / i \omega_{n}$ close to the Mott transition. At small finite doping, it reveals strong

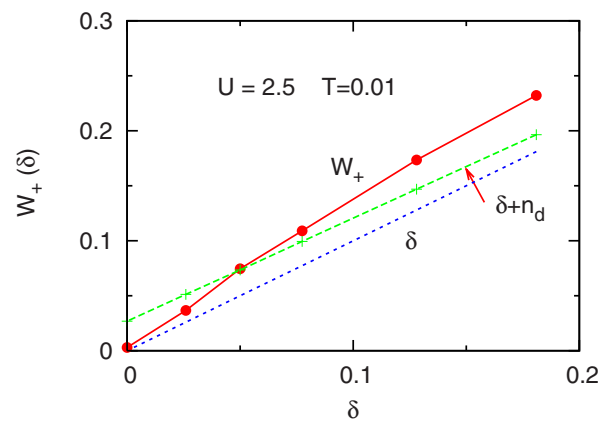

FIG. 5. (Color online) Doping-induced spectral weight $W_{+}(\delta)$ above $E_{F}=0$ up to about $\omega=0.9$, i.e., in the lower part of the main gap. A small constant weight is subtracted to account for the artificial broadening of the spectral peaks. This constant is chosen so that $W_{+}=0$ at $\mu=0.7, \delta=0$. The short-dashed blue line defines $\delta$ and the long-dashed green line $\delta+n_{d}$, where $n_{d}$ is the average double occupancy shown in Fig. 3. 



FIG. 6. (Color online) Imaginary part of self-energy molecular-orbital components $\Sigma_{m}\left(i \omega_{n}\right)$ as functions of Matsubara frequency for various chemical potentials: $\mu=0.2,0.3,0.4,0.5,0.55,0.6,0.65$ (from top to bottom) corresponding to $\delta$ $=0.24,0.18,0.12,0.08,0.05,0.03,0.01$, respectively; $U=2.5, T=0.01$.

effective-mass enhancement. Both $\operatorname{Im} \Sigma_{\Gamma}$ and $\operatorname{Im} \Sigma_{M}$ extrapolate to very small finite values in the limit $\omega_{n} \rightarrow 0$, except near the Mott transition. In striking contrast to these orbitals, $\operatorname{Im} \Sigma_{X}\left(i \omega_{n}\right)$ exhibits a finite onset in the lowfrequency limit once the doping is smaller than about $20 \%$ (see expanded scale in Fig. 7). The onset is largest at about $\mu=0.55$, corresponding to $\delta=5 \%$. At smaller doping (larger $\mu)$, i.e., very close to the Mott transition, it diminishes again.

In addition to the low-frequency onset of $\operatorname{Im~} \Sigma_{X}\left(i \omega_{n}\right)$, which gives rise to reduced quasiparticle lifetime, the nonFermi-liquid behavior also leads to a sharp initial rise and subsequent flattening of $\operatorname{Im} \Sigma_{X}\left(i \omega_{n}\right)$, which induces a sharp resonance in $\operatorname{Im} \Sigma_{X}(\omega)$ at small positive frequencies. As will be discussed in the next subsection, it is this resonance that is responsible for the pseudogap in the density of states.

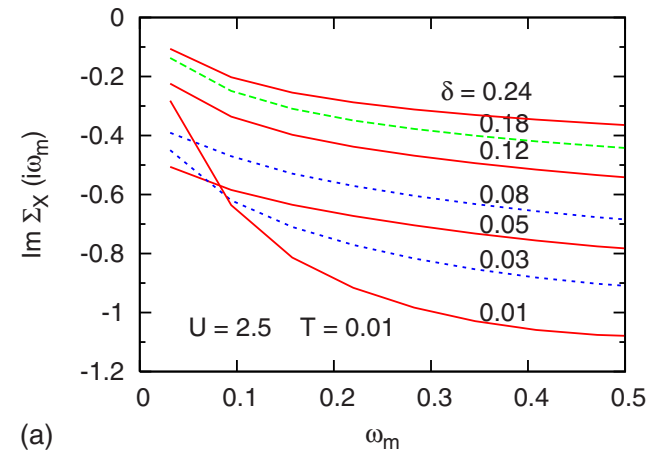

Similar results are obtained at lower temperature, $T$ $=0.005$, as shown in Fig. 7(b). Again, the largest deviation from Fermi-liquid behavior is found for $\operatorname{Im} \Sigma_{X}\left(i \omega_{n}\right)$ at about $6 \%$ doping. The onset of non-Fermi-liquid properties occurs at slightly smaller doping than for $T=0.01$. The results shown in Figs. 6 and 7 are consistent with the $T=0$ ED/ CDMFT calculations by Civelli et al. ${ }^{41}$

To illustrate the non-Fermi-liquid behavior of $\Sigma_{X}$ in more detail, we compare in Fig. 8(a) the low-frequency limits $\gamma_{m}$ $\equiv-\operatorname{Im} \Sigma_{m}\left(i \omega_{n} \rightarrow 0\right)$ as functions of chemical potential. These values were found to be nearly the same for a linear extrapolation from the first two Matsubara points and for a quadratic fit using the first three points. At $\mu>0.3$ or $\delta<0.18, \gamma_{X}$ increases strongly, indicating the onset of a non-Fermi-liquid phase. Figure 8(b) shows the variation of $\gamma_{X}$ with doping for

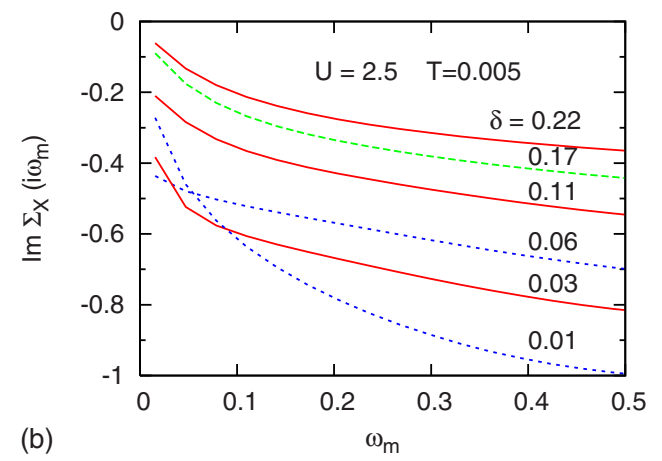

FIG. 7. (Color online) $\operatorname{Im} \Sigma_{X}\left(i \omega_{n}\right)$ for various hole-doping concentrations on expanded scale for $T=0.01$ (a) and $T=0.005$ (b). The long-dashed green curves near $\delta \approx 18 \%$ denote the approximate onset of non-Fermi-liquid behavior, which grows until $\delta$ decreases to about $5 \%$. 


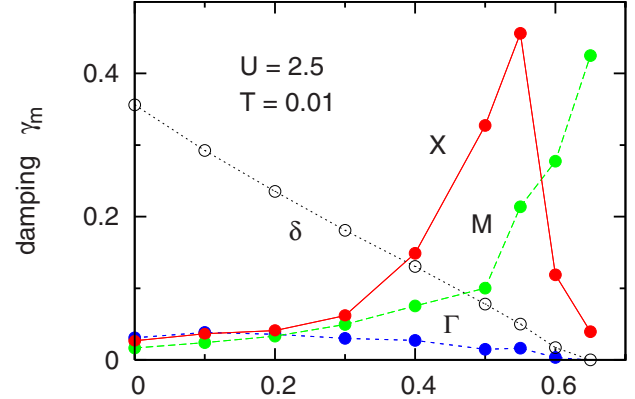

(a)

$\mu$

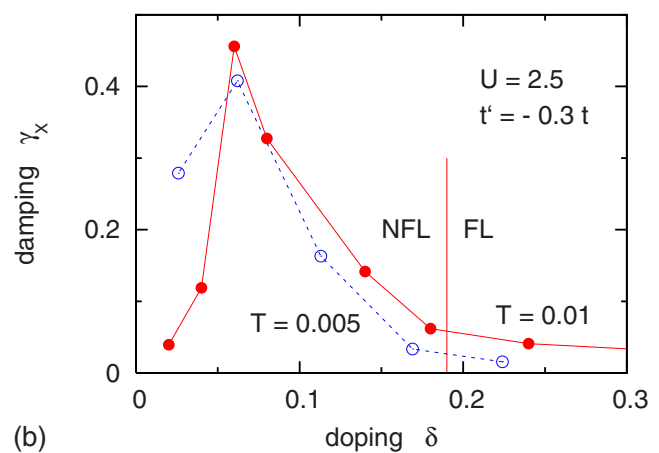

FIG. 8. (Color online) (a) Low-frequency damping rates $\gamma_{m}=-\operatorname{Im} \Sigma_{m}\left(i \omega_{n} \rightarrow 0\right)$ as functions of chemical potential. Solid red curves: $X$ orbital, short-dashed blue curves: $\Gamma$ orbital, long-dashed green curves: $M$ orbital. The dotted curve denotes the doping $\delta$ (same scale as $\gamma_{m}$ ); $U=2.5, T=0.01$. For $\mu>0.2 \ldots 0.3$ or $\delta<0.18 \ldots 0.20, \gamma_{X}$ increases strongly, indicating the onset of non-Fermi-liquid behavior. (b) Comparison of $\gamma_{X}$ as a function of doping for $T=0.01$ (solid red circles) and $T=0.005$ (empty blue circles). The vertical bar denotes the approximate location of the transition from Fermi-liquid to non-Fermi-liquid behavior.

$T=0.01$ and $T=0.005$. At lower $T$, the onset of non-Fermiliquid behavior is seen to be slightly sharper and to shift to slightly lower $\delta$.

At finite temperature, a sharp transition between Fermiliquid and non-Fermi-liquid phases is not to be expected. According to the detailed temperature variation in the selfenergy of the two-dimensional Hubbard model studied recently by Vidhyadhiraja et $a .^{64}$ within QMC/DCA for 4 $\times 4$ clusters $\left(U=1.5, t^{\prime}=0\right)$, a quantum critical point exhibiting marginal Fermi-liquid behavior was found at $\delta_{c} \approx 15 \%$ doping, with Fermi-liquid behavior at larger $\delta$ and a pseudogap phase at $\delta<\delta_{c}$. At $T=0.01$, the $T / \delta$ phase diagram indicates a crossover region of about $\delta=0.15 \pm 0.02$ between these phases. Assuming a crossover region of similar width, i.e., $\Delta \delta \approx 0.04$, the results shown in Fig. 8 are consistent with those of Ref. 64. Thus, for $U=2.5$, the Fermiliquid and non-Fermi-liquid phases seem to be separated by a quantum critical point at $\delta_{c} \approx 18 \ldots 20 \%$, with marginal Fermi-liquid behavior for $T \geq 0$.

Figure 9 compares the low-frequency damping rate of the $X$ orbital as a function of doping for $U=2.5$ and $U=1.5$. Figure 9(a) shows the results for $t^{\prime}=-0.075$, Fig. 9(b) for $t^{\prime}=0$. The case $U=1.5, t^{\prime}=0$ suggests a critical doping $\delta_{c}$ $\approx 0.15 \pm 0.02$, in agreement with the results of Ref. 64 . As is to be expected, at smaller $U \delta_{c}$ is smaller than at large $U$, since the Fermi liquid properties are stabilized. A similar trend occurs as $t^{\prime}$ is shifted from $t^{\prime}=-0.075$ to $t^{\prime}=0$. Never- theless, despite the large variations in $U$ and $t^{\prime}$, the critical doping separating the Fermi-liquid and non-Fermi-liquid phases is remarkably stable and occurs in the range of $\delta_{c}$ $\approx 0.15 \ldots 0.20$, i.e., close to the optimal doping concentrations found in many high- $T_{c}$ cuprates.

We point out here that close to the Mott transition the low-frequency damping rate $\gamma_{X}$ decreases at large $U$ but increases at small $U$ (see Fig. 9). Since we are here primarily concerned with the Fermi-liquid to non-Fermi-liquid transition, we do not discuss the limit $\delta \rightarrow 0$ in more detail. We only recall that in CDMFT all self-energy components are coupled, as indicated in Eq. (17). Thus, all molecular orbitals of the $2 \times 2$ cluster are involved in the metal-insulator transition, as shown in Figs. 3 and 4. The damping rate $\gamma_{X}$, therefore, cannot be used as the sole criterion of the Mott transition.

\section{Comparison between CDMFT and DCA}

To analyze the difference between CDMFT and DCA for $2 \times 2$ clusters, with identical system parameters, we compare in Fig. 10 the low-frequency damping rate of $\Sigma_{X}\left(i \omega_{n}\right)$ as a function of doping. Evidently, the different relations between self-energy components $\Sigma_{m}$ and lattice Green's function $G_{m}$ in these two schemes give rise to changes on a quantitative level. Nevertheless, both approaches predict a transition from a Fermi-liquid phase at hole doping larger than about $20 \%$ to
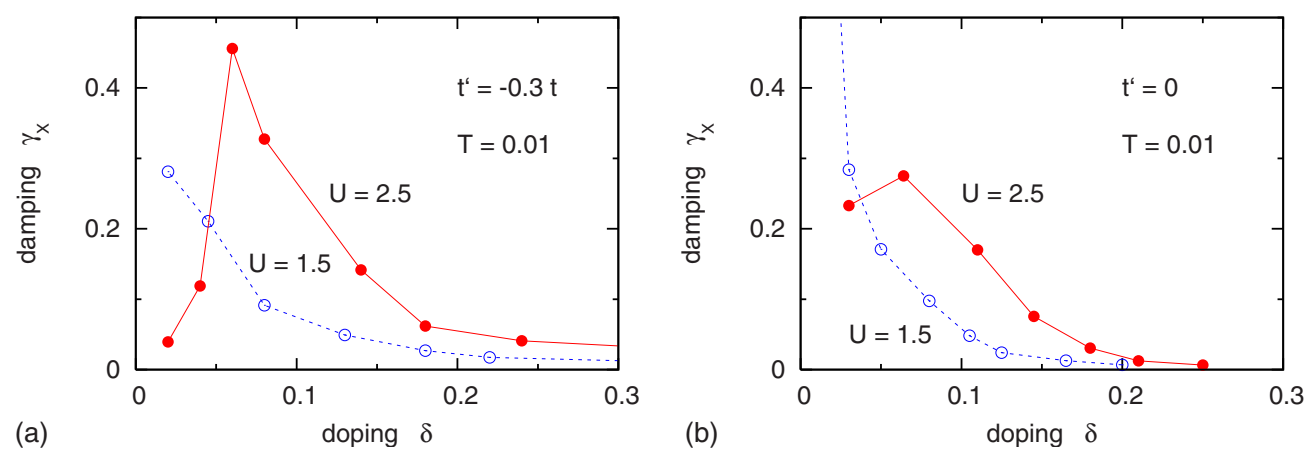

FIG. 9. (Color online) Low-frequency damping rate of $X$ orbital, $\gamma_{X}=-\operatorname{Im} \Sigma_{X}\left(i \omega_{n} \rightarrow 0\right)$ as a function of doping for various Coulomb energies and NNN hopping energies; $T=0.01$. (a) $t^{\prime}=-0.075$, (b) $t^{\prime}=0$. Solid red circles: $U=2.5$, empty blue circles: $U=1.5$. 


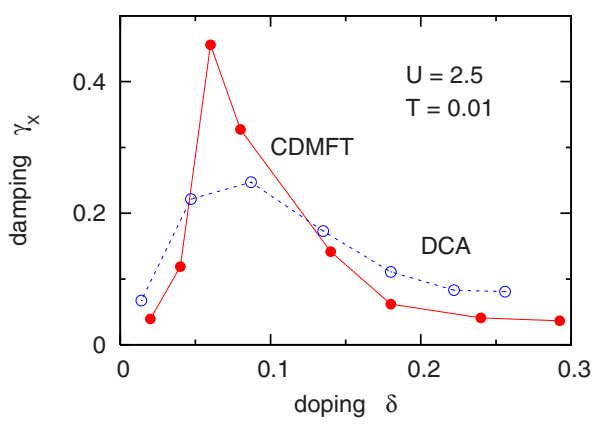

FIG. 10. (Color online) Comparison of low-frequency damping rate of $X$ orbital, $\gamma_{X}=-\operatorname{Im} \Sigma_{X}\left(i \omega_{n} \rightarrow 0\right)$ as a function of doping for CMDFT (solid red circles) and DCA (empty blue circles); $U=2.5$, $T=0.01$.

a non-Fermi-liquid phase at small doping. Surprisingly, the transition is less sharp in DCA than in CDMFT. The reason for this difference might be that, in contrast to CDMFT, the momentum patches of the Brillouin zone are not coupled in the evaluation of the DCA lattice Green's function [see Eq. (18)]. It might, therefore, be necessary in DCA to treat larger clusters (such as eight sites ${ }^{66}$ or 16 sites $^{64}$ ) in order to obtain a sharper Fermi-liquid to non-Fermi-liquid transition. A slower convergence with cluster size in DCA is also found for the critical Coulomb energy at half-filling. ${ }^{59,66}$

For a more detailed comparison with the results of Ref. 64, we show in Fig. 11 the variation in $\operatorname{Im} \Sigma_{X}\left(i \omega_{n}\right)$ with chemical potential for $U=1.5, t^{\prime}=0$, and $T=0.01$. These values of $\mu$ correspond to dopings in the range of $\delta$ $=0.27 \ldots 0.05$. Although the overall magnitude of $\operatorname{Im} \Sigma_{X}$ is much smaller than in Fig. 7 for $U=2.5, t^{\prime}=-0.075$, there is again a clear separation between doping larger than $\delta_{c}$ $\approx 0.17$ exhibiting Fermi-liquid behavior, and smaller doping with characteristic non-Fermi-liquid features. Figure 11(b) shows the comparison of the approximate quasiparticle weight, $Z_{X}=1 /\left[1-\operatorname{Im} \Sigma_{X}\left(i \omega_{0}\right) / \omega_{0}\right]$, derived from the ED/ DMFT results in Fig. 11(a), with the corresponding QMC/ DCA values taken from Fig. 1 of Ref. 64. For $\delta>0.15$ the agreement is very good. [Note that for $Z_{X}>0.5,-\operatorname{Im} \Sigma_{X}\left(i \omega_{0}\right)$ is less than $\omega_{0}=0.031$.] At smaller doping, the difference becomes larger, presumably because of the finer momentum resolution achieved for the $4 \times 4$ cluster in Ref. 64 .

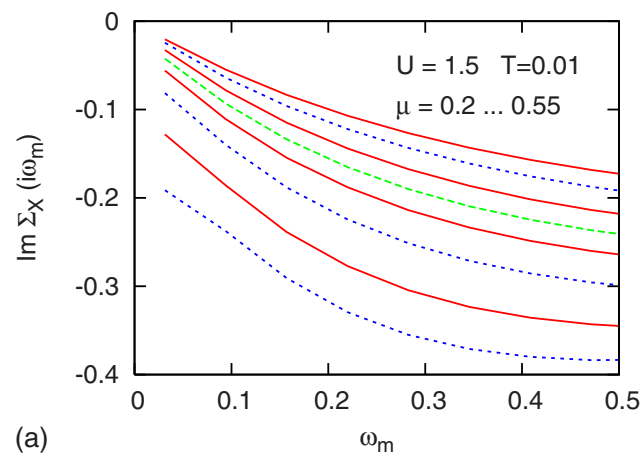

\section{Pseudogap}

The non-Fermi-liquid properties of $\Sigma_{X}\left(i \omega_{0}\right)$ manifest themselves not only in the enhanced low-frequency damping rate discussed above but also in the sharp initial rise and subsequent flattening of $\operatorname{Im} \Sigma_{X}\left(i \omega_{n}\right)$, which can be identified as the origin of the pseudogap in the density of states. Narrow gaps near $E_{F}$ below the critical doping are already evident in the cluster spectra shown in Fig. 4. Figure 12 shows these spectra on an expanded scale for $\delta=\delta_{c} \approx 0.18$ and $\delta$ $=0.03$. While near critical doping the density of states is Fermi-liquid-type, with a sharp peak at $E_{F}$, smaller hole doping leads to a very asymmetric density of states, with a pseudogap of magnitude $\Delta \approx 4 t^{2} / U=0.1$ right above $E_{F}$. The molecular-orbital analysis of these spectra reveals that this pseudogap is associated entirely with the $A_{X}(\omega)$ contribution, i.e., with the scattering processes involving momenta close to $(\pi, 0)$ and $(0, \pi)$. With decreasing doping, the peak at $E_{F}$ seen for $\delta \approx \delta_{c}$ shifts downward, so that the Fermi level gradually moves into the pseudogap. At the same time, the pseudogap becomes wider and the spectral weight above $E_{F}$ is reduced until the transition to the Mott phase occurs at half-filling. (The peak at $\omega \approx 0.25$ for $\delta=0.18$ is due to the discreteness of the cluster spectra and is not related to the pseudogap. The actual pseudogap at this large doping is vanishingly small; see analysis of self-energy below.)

Note that the peak at $E_{F}$ for $\delta=\delta_{c}$ is also compatible with marginal Fermi-liquid behavior. Finite-size effects, however, do not permit a clear distinction between Fermi-liquid properties below the first Matsubara frequency $\omega_{0}$ and genuine marginal Fermi-liquid behavior at $\delta_{c}{ }^{64}$

Figure 12(b) shows the corresponding spectra derived from the lattice Green's function components $G_{m}\left(i \omega_{n}\right)$, Eq. $(17)$, via extrapolation to real $\omega$. Thus, $A(\omega)=-\frac{1}{\pi} \operatorname{Im}\left[G_{\Gamma}(\omega)\right.$ $\left.+G_{M}(\omega)+2 G_{X}(\omega)\right] / 4$. We use here the routine ratint. ${ }^{76}$ Nearly identical spectra are obtained via Padé extrapolation. About 400...600 Matsubara points are taken into account for the energy window $-1 \leq \omega \leq 1$, and the same broadening is assumed $(\gamma=0.02)$ as in the cluster spectra shown in Fig. 12(a). As a result of the accurate self-energies and Green's functions along the Matsubara axis, the extrapolation to low real $\omega$ is highly reliable. The lattice spectra confirm the trend observed in the cluster spectra: at $\mu=0.3, \delta=0.18$, the den-

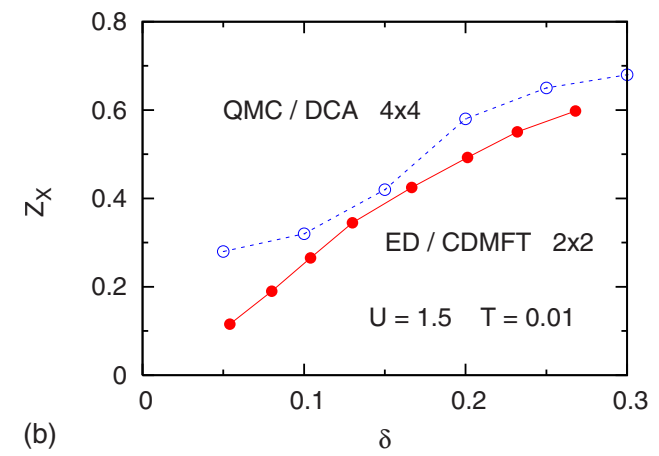

FIG. 11. (Color online) (a) Imaginary part of $\Sigma_{X}\left(i \omega_{n}\right)$ as a function of Matsubara frequency for $\mu=0.2 \ldots 0.55$ in steps of 0.05 (from top), for $U=1.5, t^{\prime}=0$, and $T=0.01 . \mu=0.3$ (long-dashed green curve) corresponds to $\delta=0.18$ and approximately marks the transition from Fermi-liquid to non-Fermi-liquid behavior. (b) Solid red circles: $Z_{X}=1 /\left(1-\operatorname{Im} \Sigma_{X}\left(i \omega_{0}\right) / \omega_{0}\right)$ derived from ED/CDMFT results in (a); empty blue circles: analogous QMC/DCA results from Fig. 1 of Ref. 64 for $T=0.014$. 

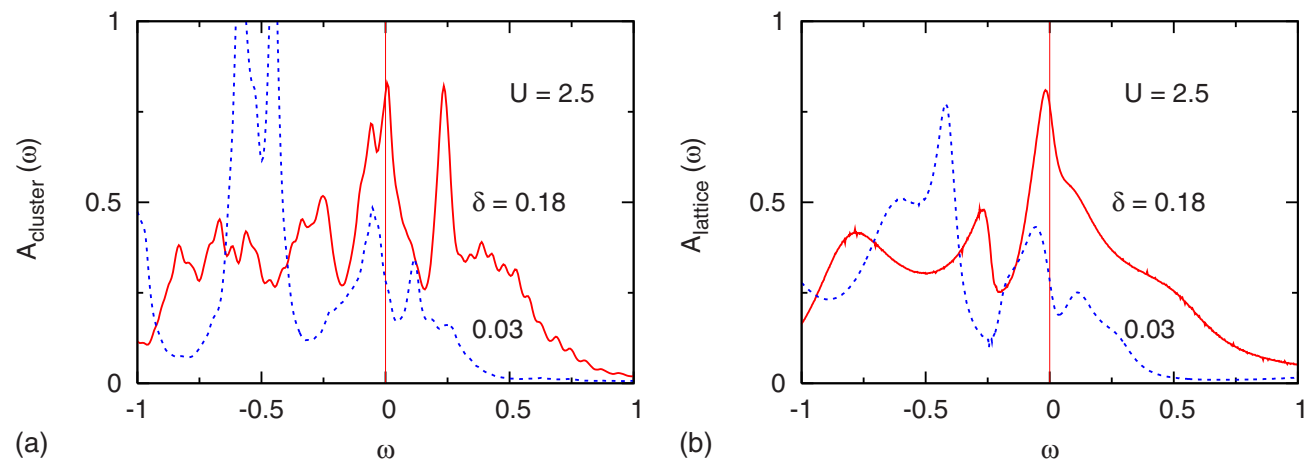

FIG. 12. (Color online) (a) Cluster spectral distributions $A(\omega)=\frac{1}{4}\left[A_{\Gamma}(\omega)+A_{M}(\omega)+2 A_{X}(\omega)\right]$ for $U=2.5, T=0.01$. Solid red curve: $\mu$ $=0.3$ (hole doping $\delta=0.18$ ) with peak at $E_{F}=0$; dashed blue curve: $\mu=0.6(\delta=0.03)$ exhibiting a pseudogap above $E_{F}$. (b) Analogous lattice spectra obtained via extrapolation of Green's function components, Eq. (17).

sity of states has a peak very close to the Fermi level, while for $\mu=0.6, \delta=0.03, E_{F}$ lies in a pseudogap of about the same width as in the cluster data. The lattice spectra $A(\omega)$ can also be calculated by first extrapolating the self-energy components $\Sigma_{m}\left(i \omega_{n}\right)$ to real frequencies and then using Eq. (17) at real $\omega$. The results are fully consistent with the spectra derived via extrapolation of $G_{m}\left(i \omega_{n}\right)$.

The pseudogap seen in Fig. 12 for $\delta=0.03$ is reminiscent of the pseudogap obtained in the two-band model within local DMFT above the first Mott transition. ${ }^{77}$ Once the electrons in the narrow subband are Mott localized, an effective two-fluid system is realized in which the Coulomb interaction with the remaining conduction electrons generates deviations from Fermi-liquid behavior, in particular, the finite lifetime associated with the low-frequency limit of $\operatorname{Im} \Sigma\left(i \omega_{n}\right)$, and the characteristic flattening of this function, which gives rise to a pseudogap at real $\omega .{ }^{78}$ This two-band model exhibits a quantum critical point when the pseudogap turns into the Mott gap. ${ }^{79}$ It would be interesting to inquire whether the present cluster picture of the single-band model could be mapped onto this two-band model. The spatial degrees of freedom in the cluster would then play the role of the interorbital fluctuations in the two-band model. Since at small hole doping a sizable number of electrons is Mott localized their spins act as scattering centers for the remaining electrons, whose self-energy then exhibits deviations from Fermi-liquid behavior.

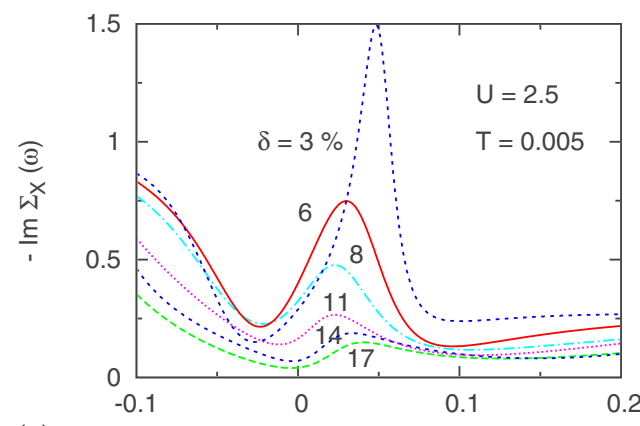

(a)

\section{E. Low-energy collective mode}

To illustrate the effect of non-Fermi-liquid behavior on the self-energy at real $\omega$, we show in Fig. 13 the lowfrequency variation in $\operatorname{Im} \Sigma_{X}(\omega)$ obtained from Fig. 7(b) via extrapolation to real $\omega$. Typically, at these low frequencies we use the first $100 \ldots 400$ Matsubara points and evaluate $\operatorname{Im} \Sigma_{X}$ at $\omega+i \gamma$, with $\gamma=0.005$. Although the details of the resulting spectra differ slightly, the important qualitative features near $E_{F}$ are very stable. Spectra derived via Padé extrapolation are very similar.

As can be seen in Fig. 13, at large hole doping $-\operatorname{Im} \Sigma_{X}(\omega)$ has a minimum at $E_{F}$ and varies quadratically at small $\omega$, as expected for a Fermi liquid. Damping in this range is very weak. Nevertheless, even for $\delta \geq 0.17$ a small peak in $-\operatorname{Im} \Sigma_{X}(\omega)$ is found at about $\omega=0.05$ above $E_{F}$, indicating that electrons added to the system above $E_{F}$ have a reduced lifetime. With decreasing doping, this feature grows into a prominent collective mode, which eventually dominates the low-frequency properties. The minimum of $-\operatorname{Im} \Sigma_{X}(\omega)$ is then shifted slightly below $E_{F}$ and a second minimum appears above $E_{F}$. $\operatorname{Re} \Sigma_{X}(\omega)$ is seen to exhibit a positive slope at the resonance, which is consistent with Kramers-Kronig relations. This implies that spectral weight is removed from the resonance region where correlation-induced damping is large. Note that, at intermediate doping near $\delta=0.14$, $\operatorname{Im} \Sigma_{X}(\omega)$ is approximately linear in $\omega$, with a larger slope above $E_{F}$ than below.

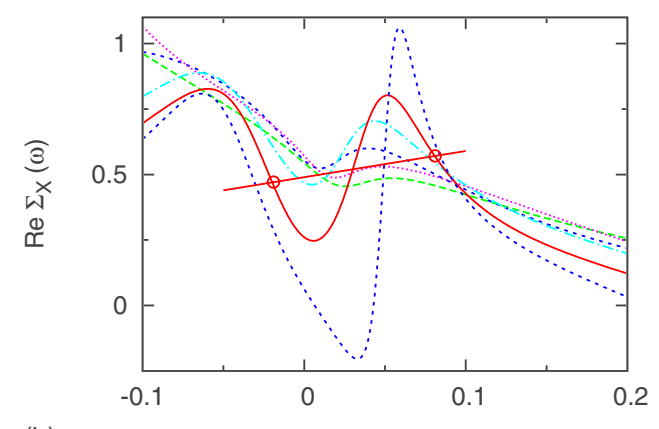

(b)

FIG. 13. (Color online) Self-energy $\Sigma_{X}(\omega)$ obtained via extrapolation to real frequencies for several hole doping concentrations (broadening $\gamma=0.005$ ). (a) $-\operatorname{Im} \Sigma_{X}$; (b) $\operatorname{Re} \Sigma_{X} ; U=2.5, T=0.005$. The outer intersections of $\operatorname{Re} \Sigma_{X}$ with the straight lines $\omega+\mu-\epsilon_{k}$ (see circles for $\delta=6 \%$ ) yield the approximate width of the pseudogap $\Delta$ plotted in Fig. 14. 

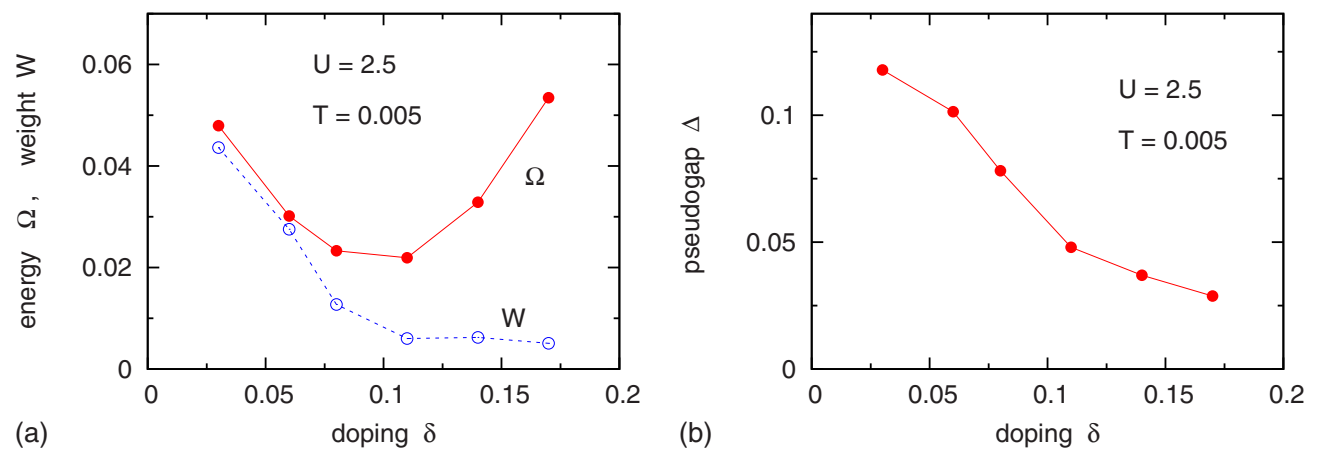

FIG. 14. (Color online) (a) Energy position $\Omega$ (solid red circles) and weight $W$ (empty blue circles) of collective mode as a function of hole doping. (b) Pseudogap $\Delta$ as a function of doping derived from self-energy $\Sigma_{X}(\omega)$.

Figure 14 shows the energy and weight (peak area) of the low-energy collective mode as a function of hole doping. The energy $\Omega$ first shifts downward from $\omega \approx 0.05$ to 0.02 and then again upward to about $\omega \approx 0.05$ at very low doping. The amplitude of this peak increases about one order of magnitude as $\delta$ decreases from 0.15 to 0.03 . The evolution of this mode with doping is one of the main results of this work and has to our knowledge not been discussed previously. A weak resonance in $\operatorname{Im} \Sigma_{X}$ at $5 \%$ doping was also found by Jarrell et $a l .{ }^{30}$ within QMC/DCA for $n_{c}=4$ (see also Ref. 46). The fact that this resonance is much stronger in the present results might be related to the faster convergence of CDMFT with cluster size (see the discussion of Fig. 10), and to the lower temperature used here. A resonance in $\operatorname{Im} \Sigma(\omega)$ is also obtained in the spectral weight transfer model proposed by Phillips et al. ${ }^{16}$ In this scheme, however, the resonance is located at $\omega=0$ independently of doping.

The outer intersections of $\operatorname{Re} \Sigma_{X}(\omega)$ with $\omega+\mu-\epsilon_{k}$ provide the approximate width of the pseudogap $\Delta$ in the spectral distribution. The central intersection does not yield any peak because of the short lifetime in this frequency range. The new minima of $-\operatorname{Im} \Sigma_{X}(\omega)$ below and above the resonance are consistent with the spectral peaks just below and above $E_{F}$, as seen in the results for $\delta=0.03$ in Fig. 12. For increasing hole doping, the resonance of $\operatorname{Im} \Sigma_{X}(\omega)$ becomes weaker so that for $\delta>0.17$ there are no longer three intersections of $\omega+\mu-\epsilon_{k}$ with $\operatorname{Re} \Sigma_{X}(\omega)$. The pseudogap then vanishes. At smaller doping, the peak in $-\operatorname{Im} \Sigma_{X}(\omega)$ grows and the pseudogap gets wider. This trend, however, is superceded by the reduction in spectral weight above $E_{F}$ as the Mott transition at half-filling is approached.

Figure 14(b) shows the approximate width of the pseudogap $\Delta$ as a function of doping. We use here the outer intersections of $\operatorname{Re} \Sigma_{X}(\omega)$ with the lines $\omega+\mu-\epsilon_{k}$ to define the magnitude of $\Delta$, where $\epsilon_{k}$ is chosen so that $\omega+\mu-\epsilon_{k}$ passes through the inflection point in the region of the maximal positive slope of $\operatorname{Re} \Sigma_{X}(\omega)$. Other values of $\epsilon_{k}$ yield similar values of $\Delta$. In the spectral distributions, this definition of the pseudogap roughly corresponds to the peak-topeak separation of spectral weight near the gap. Systematically smaller values of $\Delta$ are obtained, for instance, if the width of the gap, half way between the minimum of $A(\omega)$ and the neighboring maxima, is chosen as definition. At $\delta>0.17$, the definition used above no longer yields a pseudogap and the system turns into an ordinary Fermi liquid.
It would be very interesting to inquire to what extent the doping variation of the energy and strength of the collective mode discussed above is compatible with other theoretical models. $^{3-16}$

The doping-dependent resonance in $\operatorname{Im} \Sigma_{X}(\omega)$ and the concomitant opening of the pseudogap are consistent with recent angle-resolved photoemission (ARPES) data by Yang et al. ${ }^{71}$ According to Fig. 13(a), for $\delta \geq 0.17$ the quasiparticle damping close to $E_{F}$ is symmetric for electron and hole states. Im $\Sigma_{X}$ becomes asymmetric only for $\omega>0.02$. Below this doping, the lifetime of electron states above $E_{F}$ is much shorter than that of hole states below $E_{F}$, giving rise to the opening of the pseudogap above $E_{F}$ and the striking particlehole asymmetry observed in the data. Moreover, the results shown in Fig. 13 are specific to the $(\pi, 0)$ component of the self-energy and are absent in $\Sigma_{\Gamma}(\omega)$ and $\Sigma_{M}(\omega)$. Thus, the particle-hole asymmetry and pseudogap above $E_{F}$ are momentum-dependent features, which are most pronounced in the antinodal region, but weak or absent along the nodal $\Gamma M$ direction. This also agrees with the experimental data. ${ }^{71}$ A more detailed discussion of the momentum variation in the self-energy will be given in the final subsection.

Because of the finite temperature in the ED/CDMFT calculation, it is not possible to identify spectral features at frequencies below the first Matsubara point $\left(\omega_{0}=0.0314\right.$ for $T=0.01)$. Nevertheless, the doping variation in the pseudogap shown in Fig. 14 is found to be robust. In particular, it is clear that the pseudogap is directly linked to the resonance in $-\operatorname{Im} \Sigma_{X}(\omega)$ which, in turn, reflects the nonFermi-liquid properties of the system. Since for $\delta>\delta_{c}$ ordinary Fermi-liquid behavior is established, it is evident that the pseudogap then vanishes.

The above scenario is consistent with the fact that for a hole-doped Mott insulator the addition of electrons pushes the system closer to the insulating phase. This implies that spectral weight just above $E_{F}$ must be removed and shifted toward the upper and lower Hubbard bands. This is precisely the effect induced via the large damping associated with the low-frequency resonance in $-\operatorname{Im} \Sigma_{X}(\omega)$ and the positive slope of $\operatorname{Re} \Sigma_{X}(\omega)$.

According to this picture, the creation of holes in an electron-doped Mott insulator also moves the system closer to the insulating phase. Thus, spectral weight from states just below $E_{F}$ must be shifted to the Hubbard bands. As discussed in the next subsection, the ED/CDMFT results confirm this 

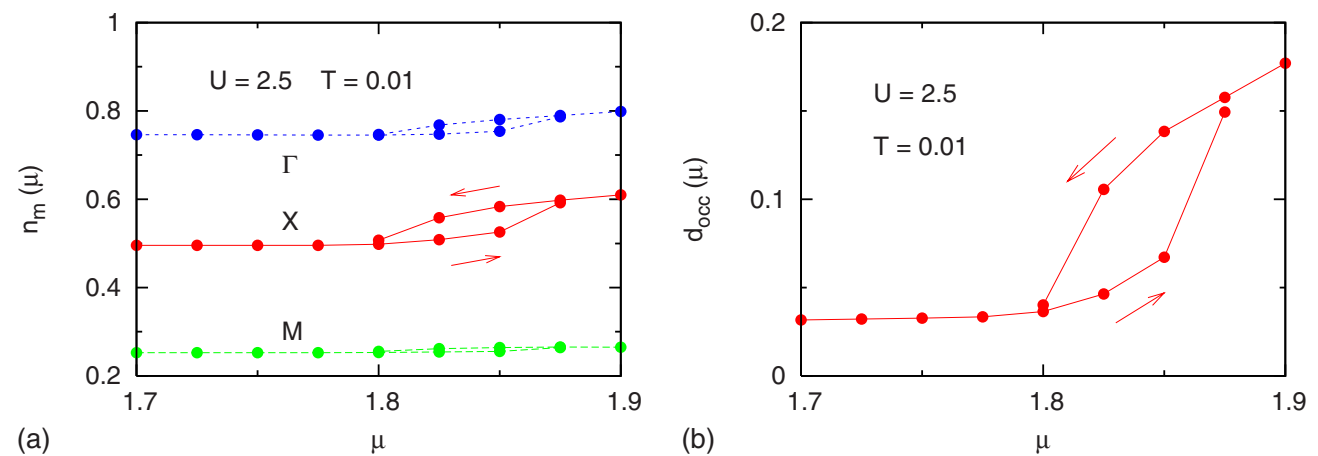

FIG. 15. (Color online) (a) Occupancies of cluster molecular orbitals (per spin) as functions of chemical potential $\mu$, for $U=2.5$, $T=0.01$. For electron doping, the Mott transition occurs at about $\mu \approx 1.8 \ldots 1.875$. In the insulating phase $0.7<\mu<1.8, n_{X}=0.5, n_{\Gamma}=0.75$, and $n_{M}=0.25$ (see also Fig. 3). The arrows denote the hysteresis behavior for increasing vs decreasing $\mu$. (b) Average double occupancy per site as a function of $\mu$.

prediction. The $X$ component of the self-energy along the Matsubara axis again exhibits non-Fermi-liquid behavior at sufficiently low electron doping. The extrapolation to real $\omega$, however, now reveals a resonance slightly below $E_{F}$, rather than above $E_{F}$ as for hole doping.

\section{F. Electron doping}

For completeness we discuss in this subsection the case of electron doping, which differs from hole doping because of the second-neighbor hopping term $t^{\prime}$. As a result of this interaction, the density of states shown in Fig. 1 is asymmetric, so that electron doping shifts the van Hove singularity away from $E_{F}$ rather than toward it. Thus, the density of states is reduced and less steep. Figure 15 shows the occupancies of the cluster molecular orbitals in the vicinity of the Mott transition induced via electron doping. Both these occupancies as well as the double occupancy shown in Fig. 15(b) exhibit hysteresis behavior for increasing vs decreasing chemical potential, indicating that this transition is first order. Thus, this transition is similar to the doping-induced metal-insulator transitions found within local DMFT for single-band and multiband systems. ${ }^{80-82}$

Because of the lower and less steep density of states for electron doping, the low-frequency variation in the self- energy differs greatly from the hole doping case, as illustrated in Fig. 16. Although there is again a clear distinction between Fermi-liquid and non-Fermi-liquid behavior, the transition now occurs at considerably smaller $\delta$. While for hole doping $\delta_{c} \approx 0.18 \ldots 0.20$, for electron doping we find $\delta_{c} \approx 0.12$. Thus, the Fermi-liquid phase is stabilized.

To identify the pseudogap for electron doping, we evaluate the cluster self-energy components via extrapolation to real frequencies. Figure 17(a) shows $\Sigma_{X}(\omega)$ at small $\omega$. In this case the non-Fermi-liquid properties give rise to a resonance in $-\operatorname{Im} \Sigma_{X}(\omega)$ centered slightly below the Fermi level, indicating that the creation of hole states in an electrondoped Mott insulator implies a transfer of spectral weight from states near $E_{F}$ to the Hubbard bands. Thereby the system is brought closer to the insulating phase. Accordingly, the real part of $\Sigma_{X}(\omega)$ exhibits a positive slope close to $E_{F}$. Its intersections with $\omega+\mu-\epsilon_{k}$ can be used to define the pseudogap. For $\delta=0.08$ the gap is found to be $\Delta \approx 0.03$, i.e., only about half as large as for the hole doping case shown in Fig. 13. Figure 17(b) shows the quasiparticle distributions obtained via extrapolation of the lattice Green's function components, Eq. (17), to real $\omega$. The dominant feature at small $\omega$ is the pseudogap in the $X$ component, which is consistent with the behavior of $\Sigma(\omega)$ displayed in Fig. 17(a).

The main difference with respect to hole doping, apart from the smaller size of the pseudogap, is the fact that this
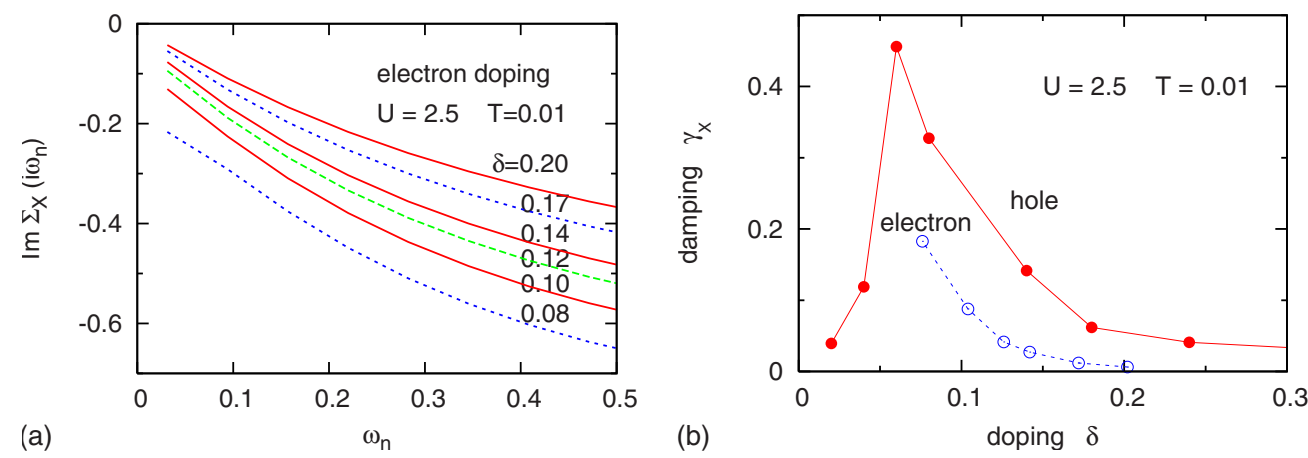

FIG. 16. (Color online) (a) Imaginary part of $\Sigma_{X}\left(i \omega_{n}\right)$ as a function of Matsubara frequency for electron doping; $U=2.5, T=0.01$. The long-dashed green curve for $\delta=0.12$ approximately marks the transition from Fermi-liquid to non-Fermi-liquid behavior. The corresponding self-energy for hole doping is shown in Fig. 7. (b) Comparison of damping $\gamma_{X}=-\operatorname{Im} \Sigma_{X}\left(i \omega_{n} \rightarrow 0\right)$ for hole doping (solid red circles) and electron doping (empty blue circles). 

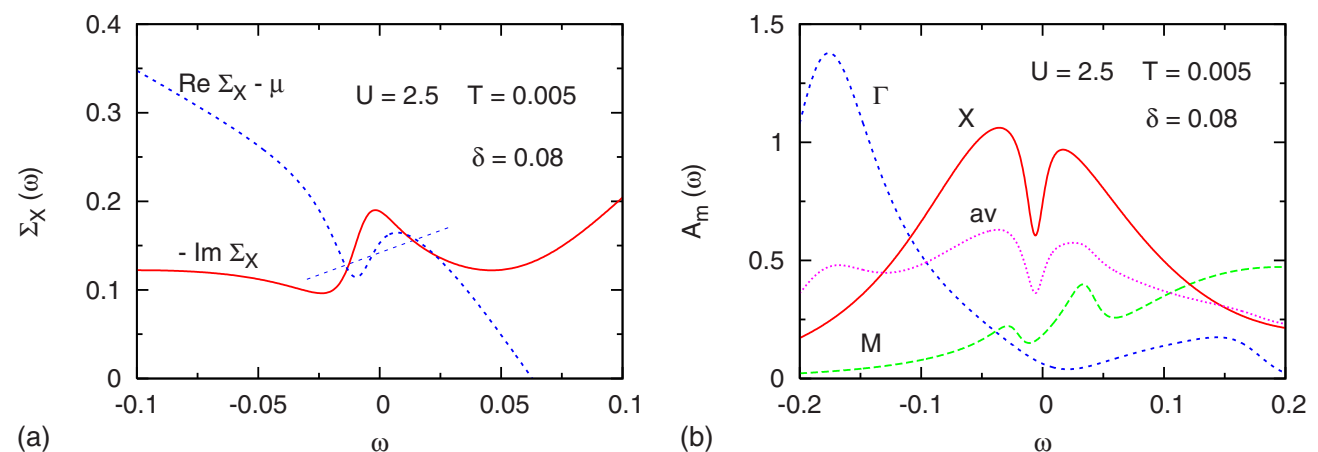

FIG. 17. (Color online) (a) Real and imaginary parts of $\Sigma_{X}(\omega)$ for electron doping obtained via extrapolation to real $\omega$. The intersections of $\operatorname{Re} \Sigma_{X}(\omega)-\mu$ with $\omega-\epsilon_{k}$ (straight line) provide the pseudogap. (b) Spectral distributions derived via extrapolation of lattice Green's function components $G_{m}\left(i \omega_{n}\right)$ to real $\omega$. The average density corresponds to $A(\omega)=-\frac{1}{4 \pi} \operatorname{Im}\left[G_{\Gamma}(\omega)+G_{M}(\omega)+2 G_{X}(\omega)\right]$.

gap can be identified only in a very narrow doping range. At electron doping larger than 0.08, the non-Fermi-liquid behavior is quickly replaced by ordinary Fermi-liquid properties. At smaller doping, spectral weight just below $E_{F}$ is rapidly transferred to the Hubbard bands, so that the pseudogap is superceded by the opening on the Mott gap.

\section{G. Phase diagram}

In Fig. 9 we have shown that the onset of non-Fermiliquid behavior is shifted to smaller hole doping when $U$ $=2.5$ is reduced to $U=1.5$ and when $t^{\prime}=-0.075$ is replaced by $t^{\prime}=0$. Figure 16 illustrates the reduction in $\delta_{c}$ for $U=2.5$ when hole doping is replaced by electron doping. A similar reduction is found for $U=1.5$ (not shown). In Fig. 18 we collect these data and display the phase diagram of the present Hubbard model for electron and hole doping. At finite temperature the values of $\delta_{c}$ can only be determined within an accuracy of about \pm 0.02 . For clarity, these margins are not plotted in Fig. 18. Despite this uncertainty, the results demonstrate several trends: for hole doping $\delta_{c}$ diminishes

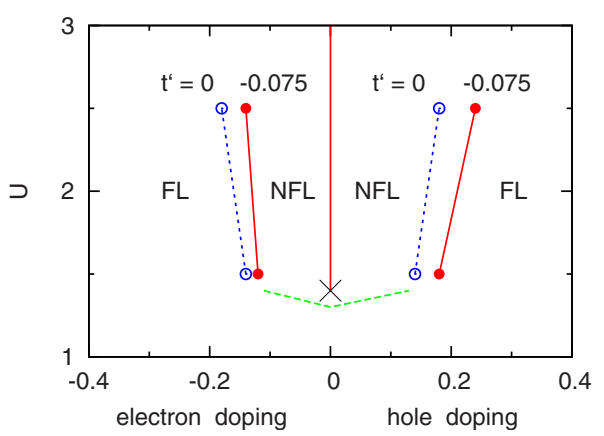

FIG. 18. (Color online) Phase diagram of 2D Hubbard model calculated within ED/CDMFT for $2 \times 2$ clusters. The Fermi-liquid phase at large hole or electron doping is turned into a non-Fermiliquid phase at small doping. The symbols for $U=1.5$ and $U=2.5$ indicate the approximate critical doping $\delta_{c}$ for $t^{\prime}=-0.075$ (solid red circles) and for $t^{\prime}=0$ (empty blue circles). The vertical line at $\delta$ $=0$ marks the Mott phase at half-filling. The critical $U$ indicated by $X$ is about 1.4 for $t^{\prime}=0$ and $t^{\prime}=-0.075$. The long-dashed green line denotes the approximate lower bound of the non-Fermi-liquid domain. with decreasing $U$ and when $t^{\prime}=-0.075$ is replaced by $t^{\prime}$ $=0$. Moreover, for $t^{\prime}=-0.075$ the critical doping decreases when hole doping is replaced by electron doping. As pointed out above, the variation in $\delta_{c}$ is surprisingly small, despite the rather large changes in $U$ and $t^{\prime}$.

\section{H. Momentum variation}

According to the results shown in Fig. 6 the non-Fermiliquid properties of the two-dimensional Hubbard model at low hole doping are mainly associated with the $X$ component of the self-energy. Only very close to the Mott transition the $M$ component begins to dominate since its imaginary part changes from $\sim \omega_{n}$ to $\sim 1 / \omega_{n}$. The cluster components of the self-energy may be used to construct an approximate momentum-dependent lattice self-energy by using the same periodization as in Eq. (19) for the Green's function. Thus, ${ }^{41}$

$$
\Sigma(\mathbf{k}, \omega)=\alpha_{\Gamma}(\mathbf{k}) \Sigma_{\Gamma}(\omega)+\alpha_{M}(\mathbf{k}) \Sigma_{M}(\omega)+\alpha_{X}(\mathbf{k}) \Sigma_{X}(\omega),
$$

where

$$
\begin{gathered}
\alpha_{\Gamma}(\mathbf{k})=\left(1+\cos k_{x}\right)\left(1+\cos k_{y}\right) / 4, \\
\alpha_{M}(\mathbf{k})=\left(1-\cos k_{x}\right)\left(1-\cos k_{y}\right) / 4, \\
\alpha_{X}(\mathbf{k})=\left(1-\cos k_{x} \cos k_{y}\right) / 2 .
\end{gathered}
$$

The k-resolved spectral distributions are then given by

$$
A(\mathbf{k}, \omega)=-\frac{1}{\pi} \operatorname{Im}[\omega+\mu-\epsilon(\mathbf{k})-\Sigma(\mathbf{k}, \omega)]^{-1} .
$$

An alternative is to periodize instead the cumulant matrix ${ }^{50}$ $M(\omega)=1 /[\omega+\mu-\Sigma(\omega)]$ which can be diagonalized in the same manner as the self-energy. Thus, the molecular-orbital components of $M(\omega)$ are given by $M_{m}(\omega)=1 /[\omega+\mu$ $\left.-\Sigma_{m}(\omega)\right]$ and the momentum-dependent lattice cumulant $M(\mathbf{k}, \omega)$ can be derived from an expression analogous to Eq. (20)

$$
M(\mathbf{k}, \omega)=\alpha_{\Gamma}(\mathbf{k}) M_{\Gamma}(\omega)+\alpha_{M}(\mathbf{k}) M_{M}(\omega)+\alpha_{X}(\mathbf{k}) M_{X}(\omega) .
$$

The lattice self-energy in this approximation takes the form 


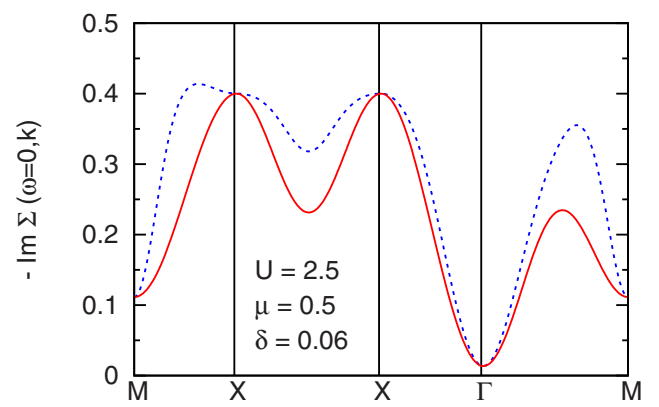

(a)

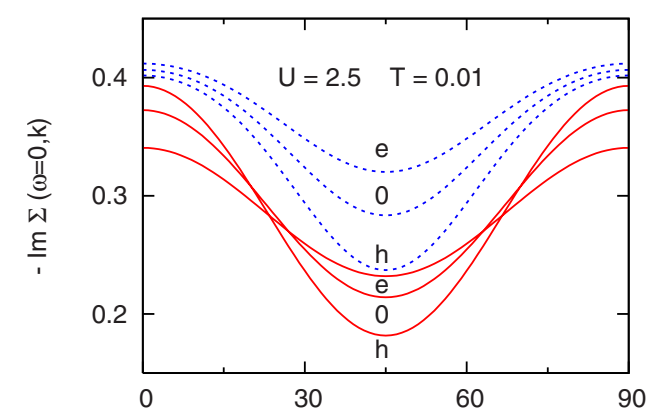

$\Phi$

FIG. 19. (Color online) (a) Momentum variation of imaginary part of $-\Sigma_{X}(\omega, \mathbf{k})$ at Fermi level. Solid red curve: expression Eq. (20), corresponding to periodization of cluster self-energy; dashed blue curve: expression Eq. (24), corresponding to periodization of cumulant; $U=2.5, \delta=0.06$, and $T=0.01$. (b) Azimuthal variation of $-\operatorname{Im} \Sigma_{X}(\omega=0, \mathbf{k})$ for $\mathbf{k}=\pi(1-r \sin \Phi, 1-r \cos \Phi)$ with $r=0.7,0.8,0.9$ (symbols $e, 0$, and $h$, respectively). $\Phi=0,90$ corresponds to $\mathbf{k}$ along $X M$ and $\Phi=45$ to the nodal direction $\Gamma M$. Solid red curves: periodization via Eq. (20); dashed blue curves: periodization via Eq. (24).

$$
\Sigma(\mathbf{k}, \omega)=\omega+\mu-1 / M(\mathbf{k}, \omega) .
$$

In Fig. 19(a) we compare these two versions of $\Sigma(\mathbf{k}, \omega)$ at $\omega=0$ for $\delta=0.06$ hole doping. The real- $\omega$ components $\Sigma_{m}(\omega=0)$ are obtained via extrapolation from the first few Matsubara frequencies. At high-symmetry points both versions of $\operatorname{Im} \Sigma(\mathbf{k}, \omega=0)$ coincide. At general $\mathbf{k}$ points, however, the cumulant expression yields enhanced damping, in particular, between $M$ and $X$, and along $\Gamma M$. The enhancement near $X$ leads to an effective flattening of $\operatorname{Im} \Sigma(\mathbf{k}, \omega)$, which is also seen in the dual Fermion approach. ${ }^{62}$ On the other hand, it is not clear whether this enhancement is partly an artifact of the cumulant approximation since the damping at some points between $X$ and $M$ is even larger than at $X$. Also, damping near $\mathbf{k} \approx 2 / 3(\pi, \pi)$ in the cumulant version is almost as large as at $X$. At the present doping $(\delta=0.06)$, the periodization of the self-energy according to Eq. (20) is in better agreement with the dual Fermion approach (see Fig. 15 of Ref. 62).

Figure 19(b) shows the variation in $-\operatorname{Im} \Sigma(\omega=0, \mathbf{k})$ along $\mathbf{k}=\pi(1-r \sin \Phi, 1-r \cos \Phi)$, where $r=0.7,0.8,0.9$ is chosen to approximately represent the region of the Fermi surface for electron doping, half-filling, and hole doping, respectively. Both periodization versions yield consistently larger damping along $X M$ than along the nodal direction $\Gamma M$. The cumulant version implies overall larger damping and,
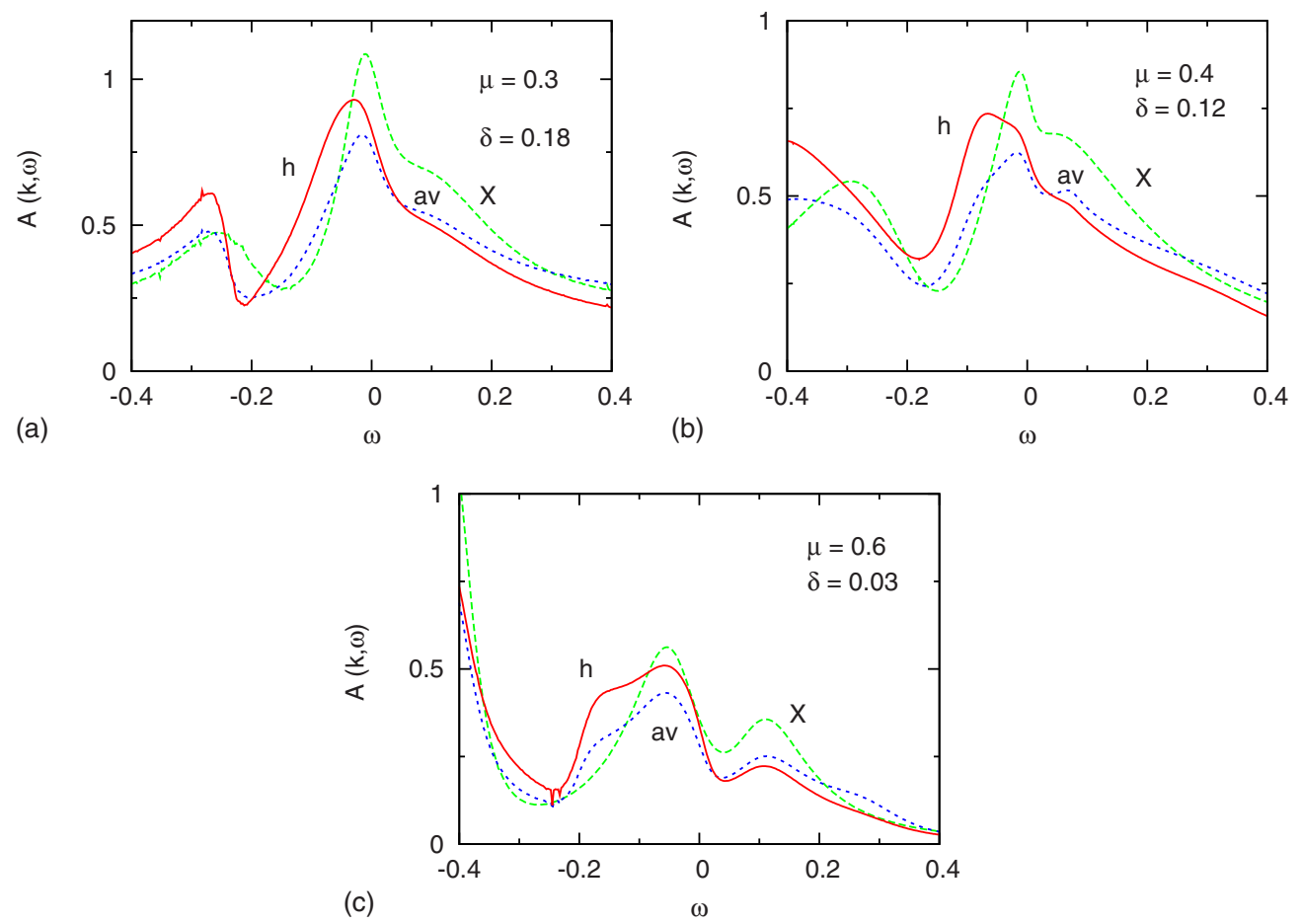

FIG. 20. (Color online) Lattice spectral distributions $A(\mathbf{k}, \omega)$ for three chemical potentials close to (a) critical doping ( $\mu=0.3$ ), (b) incipient pseudogap formation $(\mu=0.4)$, and (c) complete pseudogap phase $(\mu=0.6)$. Solid red curves $(h): k_{x}=k_{y}=0.36 \pi$ near Fermi surface for hole doping; dashed blue curves $(a v): k_{x}=k_{y}=0.5 \pi$ at center of BZ, corresponding to average density; long-dashed green curves $(X)$ : $\mathbf{k}=(\pi, 0)=X ; U=2.5, T=0.01$. 
more importantly, less pronounced difference between $\Gamma M$ and $X M$. Because of the substantial imaginary part of the self-energy at low frequencies, the Fermi surface in the present $2 \times 2$ cluster approach exhibits arcs rather than hole pockets. ${ }^{41,63}$ We emphasize, however, that greater momentum differentiation obtained for larger clusters might lead to more pronounced anisotropy between the nodal and antinodal directions. In particular, this could yield smaller values of $-\operatorname{Im} \Sigma(\omega=0, \mathbf{k})$ along $\Gamma M(\Phi=45)$ than indicated in Fig. 19.

According to the collective mode in $\operatorname{Im} \Sigma_{X}(\omega)$ (see Fig. 13), the anisotropy between the $\Gamma X$ and $\Gamma M$ directions is even larger above $E_{F}$ than for the $\omega \rightarrow 0$ limit shown in Fig. 19. Thus, the collective mode gives rise to a momentum and doping-dependent particle-hole asymmetry. To illustrate this point, we show in Fig. 20 the low-frequency part of the spectral distribution $A(\mathbf{k}, \omega)$, derived via extrapolation of the lattice Green's function, Eq. (19), at three representative points in the Brillouin zone. Three doping regions can be distinguished: at $\mu=0.3$ close to optimal doping $[\delta=0.18$, Fig. 20(a)] there is weak anisotropy since the system is a Fermi liquid throughout $\mathbf{k}$ space. Below critical doping $[\mu$ $=0.4, \delta=0.12$, Fig. 20(b)], the spectrum in the antinodal direction at $X$ shows clear signs of pseudogap behavior, while the one at $\mathbf{k}=0.36(\pi, \pi)$, i.e., near the nodal point of the Fermi surface for hole hoping, is still dominated by Fermiliquid properties. At this $\mathbf{k}$ point, the coefficients in the momentum expansion, Eq. (20), are $\left(\alpha_{\Gamma}, \alpha_{M}, \alpha_{X}\right)$ $=(0.50,0.09,0.41)$, indicating the rather large Fermi-liquidtype $\Gamma$ component. At the zone center these coefficients are $(1 / 4,1 / 4,1 / 2)$. Finally, at even lower doping $[\mu=0.6, \delta=0.03$, Fig. 20(c)], close to the Mott transition, the non-Fermi-liquid properties have spread across the entire Fermi surface, so that the pseudogap is observable along the nodal as well as antinodal directions. These results demonstrate the nonuniform, momentum-dependent opening of the pseudogap as a function of doping. (Note that this behavior differs from the opening of the Mott gap shown in Fig. 4, which in the present $2 \times 2$ cluster DMFT takes place simultaneously in all cluster components.)

\section{Comparison with ARPES data}

To make contact to the particle-hole asymmetry observed in the recent ARPES data on $\mathrm{Bi}_{2} \mathrm{Sr}_{2} \mathrm{CaCu}_{2} \mathrm{O}_{8+\delta}$ by Yang et $a l^{71}$ we have calculated the spectral distributions $A(\mathbf{k}, \omega)$ defined in Eq. (22), where the self-energy is obtained from Eq. (20). The frequency variation of the $X$ component is shown in Fig. 13. For direct comparison with the data we plot $A(\mathbf{k}, \omega)$ along three cuts, as indicated in Fig. 21. Cut 1 corresponds to the nodal direction and has the lowest relative weight from $\Sigma_{X}(\omega)$, while in cut 3 the $X$ component dominates.

For large doping $[\delta=0.17$, Fig. 22(a)], the system is a Fermi liquid. Thus, the spectral weight at all three cuts is largest at $E_{F}$ and decays symmetrically for increasing and decreasing $\omega$. Particle-hole asymmetry is then limited to energies far from $E_{F}$. Below critical doping $[\delta=0.14$, Fig. 22(b)], the particle-hole symmetry initially persists along the

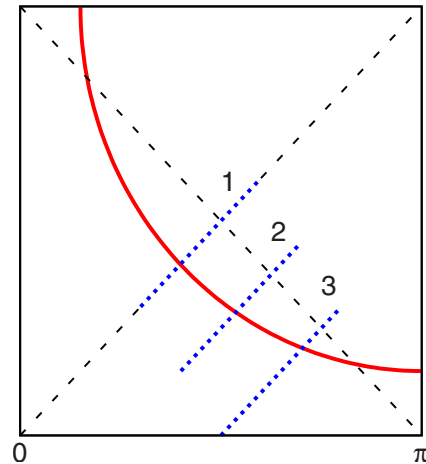

FIG. 21. (Color online) Cuts through BZ corresponding to ARPES data in Ref. 71. Solid red curve: approximate noninteracting Fermi surface for hole doping.

nodal direction, but gets weaker along cut 3 . At $\delta=0.11$ [Fig. $22(\mathrm{c})]$, this asymmetry begins to extend to the nodal direction, until at $\delta=0.08$ [Fig. 22(d)] the particle-hole asymmetry is complete throughout the Brillouin zone. These spectral distributions reveal that the particle-hole asymmetry is a direct consequence of the pseudogap, which gradually develops with doping in the region $\omega \approx 0.02 \ldots 0.05$ above $E_{F}$, and which is driven by the $(\pi, 0)$ component of the self-energy.

The momentum-dependent opening of the pseudogap above $E_{F}$, and the particle-hole asymmetry caused by the collective mode seen in $\operatorname{Im} \Sigma_{X}(\omega)$ (see Fig. 13), are in qualitative agreement with ARPES data. ${ }^{71}$ Although DMFT calculations for even larger clusters provide even better momentum differentiation, the present results for $2 \times 2$ clusters reveal that spatial degrees of freedom give rise to dramatic new phenomena absent in a local description, in particular, the collective mode in the $(\pi, 0)$ component of the selfenergy at small positive frequencies. It would be very interesting to check whether the dispersion of the position $\Omega$ of this mode with doping can be verified experimentally.

In view of the approximate nature of the momentum interpolation assumed in Eq. (20), we emphasize that the details of the spectra shown in Fig. 22 must be regarded with caution. The main points here are that (i) with decreasing doping the pseudogap moves closer to $E_{F}$ and gets wider and (ii) the origin of the pseudogap is the collective mode in $\operatorname{Im} \Sigma_{X}(\omega)$, i.e., its effects should be more pronounced near $X$ than along the nodal direction. Most likely, the difference between the nodal and antinodal directions will be even more pronounced in calculations for larger clusters. Thus, the anisotropy found here for $2 \times 2$ clusters should represent a lower bound to the actual variation between nodal and antinodal directions. Moreover, only a finer momentum resolution might allow one to distinguish between Fermi arcs and pockets.

\section{SUMMARY}

The effect of short-range correlations in the twodimensional Hubbard model is studied within finitetemperature ED combined with DMFT for $2 \times 2$ clusters. A mixed basis consisting of cluster sites and bath molecular 

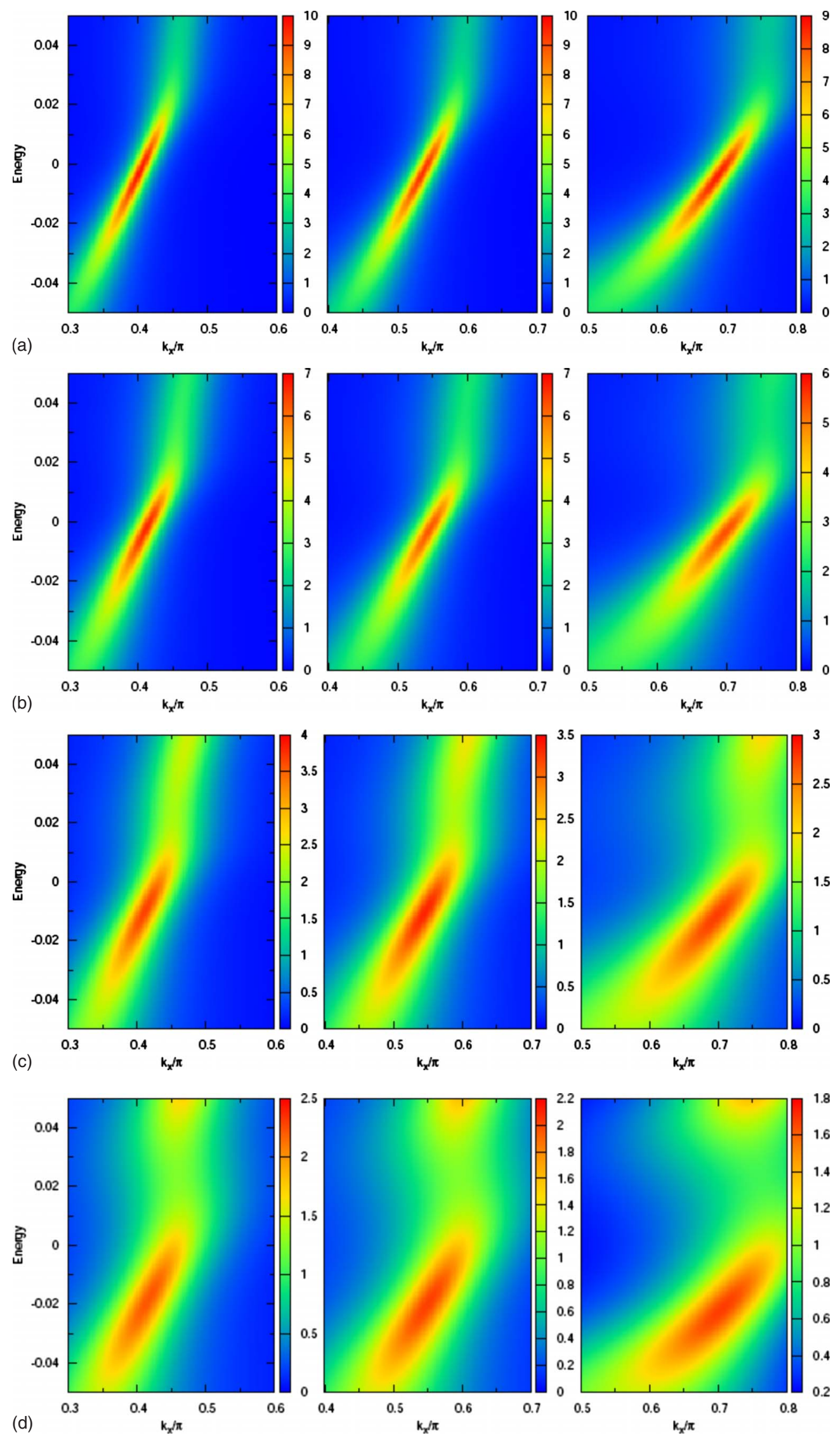

FIG. 22. (Color online) Spectral function $A(\mathbf{k}, \omega)$ along cuts 1,2 , and 3 (from left to right); (a) doping $\delta=0.17$; (b) $\delta=0.14$; (c) $\delta$ $=0.11 ;$ (d) $\delta=0.08 ; U=2.5, T=0.005$. 
orbitals is shown to provide an efficient and accurate projection of the lattice Green's function onto the cluster. To evaluate the phase diagram, the onset of non-Fermi-liquid behavior with decreasing hole doping is studied for various Coulomb energies, temperatures, and next-nearest-neighbor hopping interactions. The self-energy component $\Sigma_{X=(\pi, 0)}(\omega)$ is shown to exhibit a low-frequency collective mode above $E_{F}$, which becomes more intense close to the Mott transition. This resonance implies the removal of spectral weight from electron states above to $E_{F}$ and the opening of a pseudogap. With decreasing doping the pseudogap opens first along the antinodal direction and then spreads across the entire Fermi surface. For electron doping, the resonance of $\Sigma_{X}(\omega)$ and the corresponding pseudogap are located below $E_{F}$, as expected for the removal of hole states close to $E_{F}$. In the low-doping range the density of states at the Fermi level becomes very asymmetric. Near the onset of non-Fermi-liquid behavior, $E_{F}$ is at a maximum of the density of states. At smaller doping $E_{F}$ moves into the pseudogap. This behavior leads to a pronounced particle-hole asymmetry in the spectral distribution at intermediate hole doping, in agreement with recent ARPES measurements. The phase diagram shows that for hole doping $\delta_{c} \approx 0.15 \ldots 0.20$ for various system parameters, i.e., near the optimal doping observed in many high- $T_{c}$ cuprates. The critical electron doping, which marks the onset of non-Fermi-liquid behavior is systematically smaller than for hole doping. The Mott transition induced via electron doping exhibits first-order hysteresis characteristics. In contrast, within the present cluster ED/DMFT the hole doping transition appears to be continuous or weakly first order at very low temperatures. The consistency of these results with previous work demonstrates that finite-temperature cluster ED/ DMFT is a highly useful and accurate method that complements alternative cluster DMFT approaches.

\section{ACKNOWLEDGMENTS}

N.-H. T. is supported by the Alexander von Humboldt Foundation. The computational work was carried out on the Jülich JUMP.
${ }^{1}$ M. Imada, A. Fujimori, and Y. Tokura, Rev. Mod. Phys. 70, 1039 (1998)

${ }^{2}$ M. R. Norman, H. Ding, M. Randeria, J. C. Campuzano, T. Yokoya, T. Takeuchi, T. Takahashi, T. Mochiku, K. Kadowaki, P. Guptasarma, and D. G. Hinks, Nature (London) 392, 157 (1998).

${ }^{3}$ P. W. Anderson, G. Baskaran, Z. Zou, and T. Hsu, Phys. Rev. Lett. 58, 2790 (1987); P. W. Anderson, Science 235, 1196 (1987).

${ }^{4}$ X. G. Wen and P. A. Lee, Phys. Rev. Lett. 80, 2193 (1998).

${ }^{5}$ S. A. Kivelson, E. Fradkin, and V. J. Emery, Nature (London) 393, 550 (1998).

${ }^{6}$ C. M. Varma, Phys. Rev. Lett. 83, 3538 (1999).

${ }^{7}$ M. Vojta, Y. Zhang, and S. Sachdev, Phys. Rev. B 62, 6721 (2000).

${ }^{8}$ S. Chakravarty, R. B. Laughlin, D. K. Morr, and C. Nayak, Phys. Rev. B 63, 094503 (2001).

${ }^{9}$ C. Honerkamp, M. Salmhofer, N. Furukawa, and T. M. Rice, Phys. Rev. B 63, 035109 (2001).

${ }^{10}$ A. Läuchli, C. Honerkamp, and T. M. Rice, Phys. Rev. Lett. 92, 037006 (2004).

${ }^{11}$ R. M. Konik, T. M. Rice, and A. M. Tsvelik, Phys. Rev. Lett. 96, 086407 (2006).

${ }^{12}$ K. Y. Yang, T. M. Rice, and F. C. Zhang, Phys. Rev. B 73, 174501 (2006).

${ }^{13}$ A. M. Tsvelik and A. V. Chubukov, Phys. Rev. Lett. 98, 237001 (2007).

${ }^{14}$ S. Sachdev, Rev. Mod. Phys. 75, 913 (2003); Nat. Phys. 4, 173 (2008).

${ }^{15}$ K. Le Hur and T. M. Rice, Ann. Phys. 324, 1452 (2009).

${ }^{16}$ P. Phillips, T.-P. Choy, and R. G. Leigh, Rep. Prog. Phys. 72, 036501 (2009) and references herein; S. Chakraborty, D. Galanakis, and P. Phillips, arXiv:0807.2854 (unpublished).

${ }^{17}$ W. Metzner and D. Vollhardt, Phys. Rev. Lett. 62, 324 (1989).

${ }^{18}$ E. Müller-Hartmann, Z. Phys. B 74, 507 (1989).
${ }^{19}$ A. Georges and G. Kotliar, Phys. Rev. B 45, 6479 (1992).

${ }^{20}$ M. Jarrell, Phys. Rev. Lett. 69, 168 (1992).

${ }^{21}$ Th. Pruschke, M. Jarrell, and J. K. Freericks, Adv. Phys. 44, 187 (1995).

${ }^{22}$ A. Georges, G. Kotliar, W. Krauth, and M. J. Rozenberg, Rev. Mod. Phys. 68, 13 (1996).

${ }^{23}$ K. Held, Adv. Phys. 56, 829 (2007); G. Kotliar, S. Y. Savrasov, K. Haule, V. S. Oudovenko, O. Parcollet, and C. A. Marianetti, Rev. Mod. Phys. 78, 865 (2006).

${ }^{24}$ M. H. Hettler, A. N. Tahvildar-Zadeh, M. Jarrell, T. Pruschke, and H. R. Krishnamurthy, Phys. Rev. B 58, R7475 (1998).

${ }^{25}$ A. I. Lichtenstein and M. I. Katsnelson, Phys. Rev. B 62, R9283 (2000).

${ }^{26}$ G. Kotliar, S. Y. Savrasov, G. Palsson, and G. Biroli, Phys. Rev. Lett. 87, 186401 (2001).

${ }^{27}$ Th. A. Maier, M. Jarrell, T. Pruschke, and M. H. Hettler, Rev. Mod. Phys. 77, 1027 (2005).

${ }^{28}$ D. Sénéchal and A.-M. S. Tremblay, Phys. Rev. Lett. 92, 126401 (2004).

${ }^{29}$ M. H. Hettler, M. Mukherjee, M. Jarrell, and H. R. Krishnamurthy, Phys. Rev. B 61, 12739 (2000).

${ }^{30}$ M. Jarrell, Th. Maier, M. H. Hettler, and A. N. Tahvildarzadeh, Europhys. Lett. 56, 563 (2001); M. Jarrell, Th. A. Maier, C. Huscroft, and S. Moukouri, Phys. Rev. B 64, 195130 (2001).

${ }^{31}$ C. Huscroft, M. Jarrell, Th. A. Maier, S. Moukouri, and A. N. Tahvildarzadeh, Phys. Rev. Lett. 86, 139 (2001).

${ }^{32}$ S. Moukouri and M. Jarrell, Phys. Rev. Lett. 87, 167010 (2001).

${ }^{33}$ Y. Imai and N. Kawakami, Phys. Rev. B 65, 233103 (2002).

${ }^{34}$ Th. A. Maier, Th. Pruschke, and M. Jarrell, Phys. Rev. B 66, 075102 (2002).

${ }^{35}$ S. Onoda and M. Imada, Phys. Rev. B 67, 161102(R) (2003).

${ }^{36}$ B. Kyung, J. S. Landry, D. Poulin, and A.-M. S. Tremblay, Phys. Rev. Lett. 90, 099702 (2003).

${ }^{37}$ M. Potthoff, M. Aichhorn, and C. Dahnken, Phys. Rev. Lett. 91, 206402 (2003). 
${ }^{38}$ O. Parcollet, G. Biroli, and G. Kotliar, Phys. Rev. Lett. 92, 226402 (2004).

${ }^{39}$ M. Capone, M. Civelli, S. S. Kancharla, C. Castellani, and G. Kotliar, Phys. Rev. B 69, 195105 (2004).

${ }^{40}$ D. Sénéchal, P.-L. Lavertu, M.-A. Marois, and A.-M. S. Tremblay, Phys. Rev. Lett. 94, 156404 (2005).

${ }^{41}$ M. Civelli, M. Capone, S. S. Kancharla, O. Parcollet, and G. Kotliar, Phys. Rev. Lett. 95, 106402 (2005).

${ }^{42}$ T.-P. Choy and P. Phillips, Phys. Rev. Lett. 95, 196405 (2005).

${ }^{43}$ A. Macridin, M. Jarrell, Th. Maier, P. R. C. Kent, and E. D’Azevedo, Phys. Rev. Lett. 97, 036401 (2006).

${ }^{44}$ A.-M. S. Tremblay, B. Kyung, and D. Sénéchal, Low Temp. Phys. 32, 424 (2006).

${ }^{45}$ B. Kyung and A.-M. S. Tremblay, Phys. Rev. Lett. 97, 046402 (2006).

${ }^{46}$ B. Kyung, S. S. Kancharla, D. Sénéchal, A.-M. S. Tremblay, M. Civelli, and G. Kotliar, Phys. Rev. B 73, 165114 (2006).

${ }^{47}$ B. Kyung, G. Kotliar, and A.-M. S. Tremblay, Phys. Rev. B 73, 205106 (2006).

${ }^{48}$ M. Capone and G. Kotliar, Phys. Rev. B 74, 054513 (2006).

${ }^{49}$ T. D. Stanescu and G. Kotliar, Phys. Rev. B 74, 125110 (2006).

${ }^{50}$ T. D. Stanescu, M. Civelli, K. Haule, and G. Kotliar, Ann. Phys. 321, 1682 (2006).

${ }^{51}$ J. Merino, Phys. Rev. Lett. 99, 036404 (2007).

${ }^{52}$ A. Macridin, M. Jarrell, Th. Maier, and D. J. Scalapino, Phys. Rev. Lett. 99, 237001 (2007).

${ }^{53}$ Y. Z. Zhang and M. Imada, Phys. Rev. B 76, 045108 (2007).

${ }^{54}$ K. Haule and G. Kotliar, Phys. Rev. B 76, 104509 (2007).

${ }^{55}$ H. Park, K. Haule, and G. Kotliar, Phys. Rev. Lett. 101, 186403 (2008).

${ }^{56}$ A. Macridin and M. Jarrell, Phys. Rev. B 78, 241101(R) (2008).

${ }^{57}$ T. Senthil, Phys. Rev. B 78, 045109 (2008).

${ }^{58}$ M. Civelli, M. Capone, A. Georges, K. Haule, O. Parcollet, T. D. Stanescu, and G. Kotliar, Phys. Rev. Lett. 100, 046402 (2008).

${ }^{59}$ E. Gull, Ph. Werner, X. Wang, M. Troyer, and A. J. Millis, Europhys. Lett. 84, 37009 (2008).

${ }^{60}$ M. Balzer, B. Kyung, D. Sénéchal, A.-M. S. Tremblay, and M. Potthoff, Europhys. Lett. 85, 17002 (2009).

${ }^{61}$ M. Ferrero, P. S. Cornaglia, L. De Leo, O. Parcollet, G. Kotliar, and A. Georges, EPL 85, 57009 (2009); Phys. Rev. B 80,
064501 (2009).

${ }^{62}$ A. N. Rubtsov, M. I. Katsnelson, A. I. Lichtenstein, and A. Georges, Phys. Rev. B 79, 045133 (2009).

${ }^{63}$ S. Sakai, Y. Motome, and M. Imada, Phys. Rev. Lett. 102, 056404 (2009).

${ }^{64}$ N. S. Vidhyadhiraja, A. Macridin, C. Sen, M. Jarrell, and M. Ma, Phys. Rev. Lett. 102, 206407 (2009).

${ }^{65}$ K.-Y. Yang, H.-B. Yang, P. D. Johnson, T. M. Rice, and F.-C. Zhang, Europhys. Lett. 86, 37002 (2009).

${ }^{66}$ P. Werner, E. Gull, O. Parcollet, and A. J. Millis, Phys. Rev. B 80, 045120 (2009).

${ }^{67}$ M. Caffarel and W. Krauth, Phys. Rev. Lett. 72, 1545 (1994).

${ }^{68}$ R. B. Lehoucq, D. C. Sorensen, and C. Yang, ARPACK Users' Guide (SIAM, Philadelphia, 1997).

${ }^{69}$ A. Liebsch, H. Ishida, and J. Merino, Phys. Rev. B 78, 165123 (2008).

${ }^{70}$ A. Liebsch, H. Ishida, and J. Merino, Phys. Rev. B 79, 195108 (2009).

${ }^{71}$ H.-B. Yang, J. D. Rameau, P. D. Johnson, T. Valla, A. Tsvelik, and G. D. Gu, Nature (London) 456, 77 (2008).

${ }^{72}$ E. Koch, G. Sangiovanni, and O. Gunnarsson, Phys. Rev. B 78, 115102 (2008).

${ }^{73}$ C. A. Perroni, H. Ishida, and A. Liebsch, Phys. Rev. B 75, 045125 (2007).

${ }^{74}$ See also M. Capone, L. de' Medici, and A. Georges, Phys. Rev. B 76, 245116 (2007).

${ }^{75}$ H. Eskes, M. B. J. Meinders, and G. A. Sawatzky, Phys. Rev. Lett. 67, 1035 (1991); M. B. J. Meinders, H. Eskes, and G. A. Sawatzky, Phys. Rev. B 48, 3916 (1993).

${ }^{76}$ Numerical Recipes in Fortran 77 (Cambridge University Press, New York, 1986-1992), p. 106; J. Stoer and R. Burlisch, Introduction to Numerical Analysis (Springer, New York, 1980).

${ }^{77}$ A. Liebsch, Phys. Rev. B 70, 165103 (2004).

${ }^{78}$ A. Liebsch and T. A. Costi, Eur. Phys. J. B 51, 523 (2006).

${ }^{79}$ T. A. Costi and A. Liebsch, Phys. Rev. Lett. 99, 236404 (2007).

${ }^{80}$ G. Kotliar, S. Murthy, and M. J. Rozenberg, Phys. Rev. Lett. 89, 046401 (2002).

${ }^{81}$ D. J. García, E. Miranda, K. Hallberg, and M. J. Rozenberg, Phys. Rev. B 75, 121102(R) (2007).

${ }^{82}$ A. Liebsch, Phys. Rev. B 77, 115115 (2008). 